\title{
Surface Meshing with Curvature Convergence
}

\author{
Huibin Li, Student Member, IEEE, Wei Zeng, Member, IEEE, Jean Marie Morvan, \\ Liming Chen, Member, IEEE, and Xianfeng David Gu*, Member, IEEE
}

\begin{abstract}
Surface meshing plays a fundamental role in graphics and visualization. Many geometric processing tasks involve solving geometric PDEs on meshes. The numerical stability, convergence rates and approximation errors are largely determined by the mesh qualities. In practice, Delaunay refinement algorithms offer satisfactory solutions to high quality mesh generations. The theoretical proofs for volume based and surface based Delaunay refinement algorithms have been established, but those for conformal parameterization based ones remain wide open. This work focuses on the curvature measure convergence for the conformal parameterization based Delaunay refinement algorithms. Given a metric surface, the proposed approach triangulates its conformal uniformization domain by the planar Delaunay refinement algorithms, and produces a high quality mesh. We give explicit estimates for the Hausdorff distance, the normal deviation, and the differences in curvature measures between the surface and the mesh. In contrast to the conventional results based on volumetric Delaunay refinement, our stronger estimates are independent of the mesh structure and directly guarantee the convergence of curvature measures. Meanwhile, our result on Gaussian curvature measure is intrinsic to the Riemannian metric and independent of the embedding. In practice, our meshing algorithm is much easier to implement and much more efficient. The experimental results verified our theoretical results and demonstrated the efficiency of the meshing algorithm.
\end{abstract}

Index Terms-Meshing, Delaunay refinement, conformal parameterization, normal cycle, curvature measures, convergence.

\section{INTRODUCTION}

Surface meshing and remeshing play fundamental roles in many engineering fields, including computer graphics, geometric modeling, visualization and medical imaging. Typically, surface meshing finds a set of sample points on the surface with a curved triangulation, then approximates each face by an Euclidean triangle in $\mathbb{R}^{3}$, thereby approximating the underlying smooth surface by a polyhedral triangular surface, which is called a triangle mesh.

Many geometric processing tasks are equivalent to solving geometric partial differential equations (PDEs) on surfaces. The following are some direct examples: for shape analysis, the heat kernel signature (HKS) [1] is mostly utilized, which entails solving a heat equation and computing the eigenvalues and eigenfunctions of the Laplace-Beltrami operator on the surfaces; for shape registration, the surface harmonic map [2] is widely used, which essentially means solving elliptic PDEs on the surfaces; for surface parameterization, the discrete Ricci flow [3] is often computed, which amounts to solving a nonlinear parabolic equation on the surfaces.

Most geometric PDEs are discretized on triangle meshes, and solved using numerical methods, such as Finite Element Methods (FEM). The numerical stability, the convergence

- H. Li and L. Chen are with the Department of Mathematics and Informatics, Ecole Centrale Lyon, France.E-mail: huibin.li,liming.chen@ec-lyon.fr.

- W. Zeng is with the School of Computing and Information Sciences, Florida International University, USA. E-mail: wzeng@cs.fiu.edu.

- J. M. Morvan is with the Department of Mathematics, Institut Camille Jordan, Université Lyon 1, France, and the GMSV Research Center, King Abdullah University of Science and Technology, Saudi Arabia. E-mail: morvan@math.univ-lyon1.fr.

- * Corresponding author: X. David Gu is with the Department of Computer Science, State University of New York at Stony Brook, USA. E-mail: gu@cs.sunysb.edu.

Manuscript received November 26, 2012; revised February 18, 2013. rates, and the approximation bounds of the discrete solutions are largely determined by the quality of the underlying triangle mesh, which is measured mainly by the size and the shape of triangles on the mesh. Therefore, the generation of high quality meshes has fundamental importance.

Most existing meshing and remeshing approaches are based on the Delaunay refinement algorithms. They can be classified in three main categories:

1) The sampling is computed in $\mathbb{R}^{3}$, and triangulated using the volumetric Delaunay triangulation algorithms, such as [4] [5] [6] [7] [8] [9].

2) The sampling and triangulation are directly computed on curved surfaces, such as [10] [11].

3) The sampling is computed in a conformal parameter domain, and triangulated using the planar Delaunay triangulation algorithms, such as [12] [13] [14] [15] [16].

The convergence theories of curvature measures for the approaches in the first two categories has been thoroughly established in [17] [18] [19] [20]. However, so far, there is no theory to show the convergence of curvature measures for the approaches in the third category.

\subsection{Existing Theoretical Results}

Based on the classic results of Federer [21] and Fu [22], among others, the authors in [17] [18] [19] defined a general and unified framework of curvature measures for both smooth and discrete submanifolds of $\mathbb{R}^{N}$ based on the normal cycle theory. Furthermore, they proved the convergence and approximation theorems of curvature measures for the general geometric subset of $\mathbb{R}^{N}$.

In particular, suppose $M$ is a smooth surface embedded in $\mathbb{R}^{3}, M_{\varepsilon}$ is an $\varepsilon$-sample of $M$, namely, for each point $p \in M$, the ball $B(p, \varepsilon \operatorname{lfs}(p))$ contains at least one sample point in $M_{\varepsilon}$, 
where lfs $(p)$ denotes the local feature size of $M$ at point $p$. Let $T$ be the triangle mesh induced by the volumetric Delaunay triangulation of $M_{\varepsilon}$ restricted to $M$. If $\varepsilon$ is small enough, each point of the mesh has a unique closest point on the smooth surface. This leads to the introduction of the closest point projection $\pi: T \rightarrow M$. This map has the following properties:

1) Normal deviation: $\forall p \in T,|\mathbf{n}(p)-\mathbf{n} \circ \pi(p)|=O(\varepsilon)$, by Amenta et al. [4], and Boissonnat et al. [6].

2) Hausdorff distance: $|p-\pi(p)|=O\left(\varepsilon^{2}\right)$, by Boissonnat et al. [6].

3) Homeomorphism: $\pi$ is a global homeomorphism, by Amenta et al. [4] and Boissonnat et al. [6].

4) Curvature measures: Let $B$ be a Borel subset of $\mathbb{R}^{3}$, then the differences between the curvature measures on $M$ and those on $T$ are $K \varepsilon$, where $K$ depends on the triangulation $T$ [17] [19].

In the first category, the authors show that, unfortunately, the convergence of curvature measures can not be guaranteed. Depending on the triangulation, when $\varepsilon$ goes to $0, K$ may go to infinity, (see [18] for a counterexample). To ensure the convergence of the curvature measures, in [18] [19], the authors suggest adding a stronger assumption to the sampling condition, namely, $\kappa$-light $\varepsilon$-sample, which is an $\varepsilon$-sample with the additional constraint that each ball $B(p, \varepsilon \operatorname{lfs}(p))$ contains at most $\kappa$ sample points.

In the second category, the curvature convergence for meshes obtained by Chew's second algorithm [11] has been proved in [20]. The normal and area convergence for meshes based on the geodesic Delaunay refinement algorithm has been proved in [10]. However, the computation of the geodesic Delaunay triangulation is prohibitively expensive in practice [23].

\subsection{Our Theoretical Results}

This paper will deal with triangulations of the third category, showing stronger estimates. Using conformal parameterization, we obtain meshes satisfying the first two properties as before,

1) Normal deviation: $O(\varepsilon)$, Lemma 4.8 and Lemma 4.9.

2) Hausdorff distance: $O\left(\varepsilon^{2}\right)$, Lemma 4.8 and Lemma 4.9.

Moreover, we improve the other two properties as follows:

3) Homeomorphism: In addition to the closest point projection $\pi$, we also define a novel mapping, the natural projection $\eta$, induced by the conformal parameterization. Both projections are global homeomorphisms, see section 4.4.3.

4) Curvature measures: we show the Delaunay refinement method on the conformal parameter domain generates $\kappa$-light $\varepsilon$-sample, which guarantees the convergence of curvature measures. Moreover, we show that the bounds of the curvature measures are $K \varepsilon$, where $K$ is $O(\operatorname{area}(B))+O($ length $(\partial B))$, and are independent of the triangulations, see Theorem 3.4 and section 4.4.4.

In addition, the coding and computational complexities are much lower than those in the second category.

\subsubsection{Similarities}

Following the work in [17], our proof is mainly based on the normal cycle theory. Both methods estimate both the Hausdorff distance and the normal deviation at the corresponding points. Then both methods construct a homeomorphism from the triangle mesh to the surface, which induces a homotopy from the normal cycle of the mesh to the normal cycle of the surface. Then, the volume swept by the homotopy and the area of its boundary are estimated. This gives a bound on the difference between the curvature measures.

\subsubsection{Differences}

However our work can be clearly differentiated from theirs, in terms of both theoretical and algorithmic aspects:

- In theory, as pointed out previously, without the stronger sampling condition, the volumetric Delaunay refinement algorithms cannot guarantee the convergence of curvature measures. In contrast, our results can ensure the convergence without extra assumptions.

- In theory, the volumetric Delaunay refinement methods require the embedding of the surface. Our method is intrinsic, which only requires the Riemannian metric. In many real-life applications, e.g. the general relativity simulation in theoretical physics, the surface metric is given without any embedding space. In such cases, the volumetric Delaunay refinement methods are invalid, but our method can still apply.

- In theory, to prove the main theorem, the closest point mapping was constructed in [17]. In contrast, we supply two proofs: one is based on the closest point mapping, whereas the other uses a completely different mapping based on conformal parameterization. Conceptually, besides its novelty, the latter is also simpler.

- In practice, the planar Delaunay refinement methods are much easier to implement, the data structure for planar triangulation is much simpler than that of the tetrahedral mesh, and the planar algorithms are much more efficient.

Remark The current meshing algorithm aims to achieve a good triangulation, and requires a conformal parameterization, which in turn requires a triangulation. Consequently, this looks like a chicken-and-egg problem.

In fact, conformal parameterization can be carried out using an initial triangulation of low quality, and this algorithm will produce a new triangulation with much better quality. Many geometric processing tasks cannot be computed on the initial mesh. For example, the error bound for a discrete solution to the Poisson equation is $O\left(\varepsilon^{2}\right)$ on good quality meshes. If the mesh has too many obtuse angles, then the discrete results will not converge to the smooth solution.

In reality, surfaces are acquired by 3D scanning devices, such as the laser scanner or the structured light scanner. Usually, the raw point clouds are very dense, thus the initial triangulation can be induced by the pixel or voxel grid structures. In the geometric modeling field, the input surfaces may be spline surfaces, and the initial triangulation can be chosen as the regular grids on the parameter domain. Then, the conformal parameterizations can be computed using the 
dense samples with the initial triangulation. Finally, we can perform the remeshing using the current conformal parametric Delaunay refinement algorithm to improve the mesh quality or compress the geometric data.

\section{Previous Works}

\subsection{Meshing/Remeshing}

\subsubsection{Delaunay Refinement}

The Delaunay refinement algorithms were originally designed for meshing planar domains, and were later generalized for meshing surfaces and volumes. Chew's first algorithm [24] splits any triangle whose circumradius is greater than the prescribed shortest edge length parameter $\varepsilon$ and hence generates triangulation of uniform density and with no angle smaller than $30^{\circ}$. But the number of triangles produced is not optimal. Chew's second algorithm [11] splits any triangle whose circumradius-to-shortest-edge ratio is greater than one, and hence in practice produces grade mesh. Similar split criterion was used in Ruppert's algorithm [25], which has the theoretical guarantee of the minimal angle of no less than $20.7^{\circ}$. Shewchuk's algorithm [26] can create meshes with most angles of $30^{\circ}$ or greater. Dey et al. developed a series of algorithms for surface meshing and remeshing based on volumetric Delaunay refinement [7] [8] [9], which belong to the approaches in the first category. We refer readers to [27] for full details.

\subsubsection{Centroidal Voronoi Tessellation}

The concept of centroidal Voronoi tessellations (CVT) was first proposed by Du et al. [28], and then was generalized to constrained centroidal Voronoi tessellations (CCVT) [29]. Recently, CVT has been widely used for surface meshing/remeshing to produce high quality triangulations. It can be carried out in the ambient space, e.g. Yan et al. [30], or the conformal parameter domain, e.g. Alliez et al. [12] [31], or even high embedding space, e.g. Lévy et al. [32]. A complete survey of the recent advancements on CVT based remeshing can be found in [16]. Although visually pleasing and uniform, all the existing CVT based remeshing methods for the generation of high quality triangulation have no theoretical bound of the minimal angle [16]. Therefore, the convergence of curvature measures cannot be guaranteed.

\subsection{Conformal Surface Parameterization}

Over the last two decades, surface parameterization has gradually become a very popular tool for various mesh processing processes [33] [34]. In this work, we consider only conformal parameterizations. There are many approaches used for this purpose, including the harmonic energy minimization [35] [36] [37], the Cauchy-Riemann equation approximation [38], Laplacian operator linearization [39], circle packing [40], angle-based flattening [41], holomorphic differentials [42], Ricci curvature flow [3] [43], Yamabe flow [44], conformal equivalence class [45], most isometric parameterizations (MIPS) [46], etc. .

\section{StATEMENT OF THE MAIN THEOREM}

\subsection{Curvature Measures}

First, let $M$ be a $C^{2}$-smooth surface embedded in $\mathbb{R}^{3}$, its curvature measures can be defined as follows.

Definition 3.1: The Gaussian curvature measure of $M, \phi_{M}^{G}$, is the function associated with each Borel set $B \subset \mathbb{R}^{3}$,

$$
\phi_{M}^{G}(B)=\int_{B \cap M} G(p) d p
$$

where $G(p)$ is the Gaussian curvature of $M$ at point $p$. Similarly, the mean curvature measure $\phi_{M}^{H}$ is given by

$$
\phi_{M}^{H}(B)=\int_{B \cap M} H(p) d p
$$

where $H(p)$ denotes the mean curvature of $M$ at point $p$.

Now, let $V$ be a polyhedron of $\mathbb{R}^{3}$ and its polyhedral boundary $M$ be a triangular mesh surface. We use $v_{i}$ to denote a vertex, $\left[v_{i}, v_{j}\right]$ an edge, and $\left[v_{i}, v_{j}, v_{k}\right]$ a face of $M$. We define the discrete Gaussian curvature of $M$ at each vertex as the angle deficit,

$$
G\left(v_{i}\right)=2 \pi-\sum_{j k} \theta_{i}^{j k},
$$

where $\theta_{i}^{j k}$ is the corner angle on the face $\left[v_{i}, v_{j}, v_{k}\right]$ at the vertex $v_{i}$. Similarly, the discrete mean curvature at each edge is defined as

$$
H\left(e_{i j}\right)=\left|v_{i}-v_{j}\right| \beta\left(e_{i j}\right),
$$

where $\beta_{i j}$ is the angle between the normals to the faces incident to $e_{i j}$. The sign of $\beta\left(e_{i j}\right)$ is chosen to be positive if $e_{i j}$ is convex and negative if it is concave.

Definition 3.2: The discrete Gaussian curvature measure of $M, \phi_{M}^{G}$, is the function associated with each Borel set $B \subset \mathbb{R}^{3}$

$$
\phi_{M}^{G}(B)=\sum_{v \in B \cap M} G(v) .
$$

The discrete mean curvature measure $\phi_{M}^{H}$ is

$$
\phi_{M}^{H}(B)=\sum_{e \in B \cap M} H(e) .
$$

The curvature measures on both smooth surfaces and polyhedral surfaces can be unified by the normal cycle theory, which will be explained in section 4.3.

\subsection{Main Results}

It is well known that any Riemannian metric defined on a smooth (compact with or without boundary) surface $M$ can be conformally deformed into a metric of constant curvature $c \in\{-1,0,1\}$, depending on the topology of $M$, the so-called uniformization metric (cf. Fig. 1). Now if $M$ is endowed with a Riemannian metric with constant curvature, the Delaunay refinement algorithms can be used to generate a triangulation on $M$ with good quality.

The most common Delaunay refinement algorithms include Chew's [24], [11] and Ruppert's [25]. Let $\varepsilon$ be a user defined upper bound of the circumradius of the final triangulation. Given an initial set of samples on surface $M$, such that the distance between any pair of samples is greater than $\varepsilon$. If $M$ has boundaries, then the boundaries are sampled and approximated by piecewise geodesics, such that each 
geodesic segment is greater than $\varepsilon$. The Delaunay refinement method on the uniformization space starts with an initial Delaunay triangulation of the initial samples, then updates the samples by inserting circumcenters of the bad triangles, and meanwhile, updates the triangulation by maintaining the Delaunay property. A bad triangle can be either bad-sized or bad-shaped. A triangle is bad-sized, if its circumradius is greater than $\varepsilon$. A triangle is bad-shaped, if its circumradiusto-shortest-edge ratio is greater than one. In this work, we will show the following meshing algorithm using the packing argument.

Theorem 3.3 (Delaunay Refinement): Let $M$ be a compact Riemannian surface with constant curvature. Suppose that the boundary of $M$ is empty or is a union of geodesic circles. For any given small enough $\varepsilon>0$, the Delaunay refinement algorithm terminates. Moreover, in the resultant triangulation, all triangles are well-sized and well-shaped, that is

1) The circumradius of each triangle is not greater than $\varepsilon$.

2) The shortest edge length is greater than $\varepsilon$.

Suppose $M$ is also embedded in $\mathbb{E}^{3}$ with the induced Euclidean metric. Then $M$ can also be conformally mapped to a surface with uniformization metric, such that all boundaries (if there are any) are mapped to geodesic circles. By running the Delaunay refinement on the uniformization space, we can get a triangulation of $M$, which induces a polyhedral surface $T$, whose vertices are on the surface, and all faces of which are Euclidean triangles. Furthermore, all triangles are wellsized and well-shaped under the original induced Euclidean metric. Based on the induced triangulation $T$, we will show the following main theorem.

Theorem 3.4 (Main Theorem): Let $M$ be a compact Riemannian surface embedded in $\mathbb{E}^{3}$ with the induced Euclidean metric, $T$ the triangulation generated by Delaunay refinement on conformal uniformization domain, with a small enough circumradius bound $\varepsilon$. If $B$ is the relative interior of a union of triangles of $T$, then:

$$
\begin{aligned}
\left|\phi_{T}^{G}(B)-\phi_{M}^{G}(\pi(B))\right| & \leq K \varepsilon \\
\left|\phi_{T}^{H}(B)-\phi_{M}^{H}(\pi(B))\right| & \leq K \varepsilon \\
\left|\phi_{T}^{G}(B)-\phi_{M}^{G}(\eta(B))\right| & \leq K \varepsilon \\
\left|\phi_{T}^{H}(B)-\phi_{M}^{H}(\eta(B))\right| & \leq K \varepsilon
\end{aligned}
$$

where for fixed $M$

$$
K=O\left(\sum_{\{t \in T, t \subset \bar{B}\}} r(t)^{2}\right)+O\left(\sum_{\{t \in T, t \subset \bar{B}, t \cap \partial B \neq \emptyset\}} r(t)\right),
$$

$r(t)$ being the circumradius of triangle $t$. Moreover, $K$ can be further replaced by: $K=O(\operatorname{area}(B))+O($ length $(\partial B))$. Furthermore, if $M$ is an abstract compact Riemannian surface (only with a Riemannian metric, but not an embedding), inequalities (3) and (5) still hold.

Here $\pi$ denotes the closest point projection on $M$, and $\eta$ denotes the natural projection on $M$, which is induced by the conformal parameterization, see Definitions 4.6 and 4.7.

\section{Theoretical Proofs}

\subsection{Surface Uniformization}

Let $\left(M_{1}, \mathbf{g}_{1}\right)$ and $\left(M_{2}, \mathbf{g}_{2}\right)$ be smooth surfaces with Riemannian metrics. Let $\phi: M_{1} \rightarrow M_{2}$ be a diffeomorphism, $\phi$ is conformal if and only if

$$
\phi^{*} \mathbf{g}_{2}=e^{2 \lambda} \mathbf{g}_{1},
$$

where $\phi^{*} \mathbf{g}_{2}$ is the pullback metric on $M_{1}$, and $\lambda: M_{1} \rightarrow \mathbb{R}$ is a scalar function defined on $M_{1}$. Conformal mappings preserve angles and distort area elements. The conformal factor function $e^{2 \lambda}$ indicates the area distortion.

According to the classical surface uniformization theorem, every metric surface $(M, \mathbf{g})$ can deform to one of three canonical shapes, a sphere, a Euclidean plane or a hyperbolic plane. Namely, there exists a unique conformal factor function $\lambda: M \rightarrow \mathbb{R}$, such that the uniformization Riemannian metric $e^{2 \lambda} \mathbf{g}$ induces constant Gaussian curvature, the constant being one of $\{+1,0,-1\}$ according to the topology of the surface. If surfaces have boundaries, then the boundaries are mapped to circles on the uniformization space.
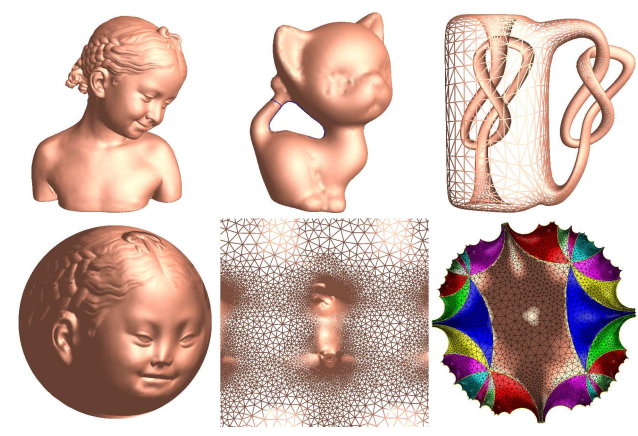

Fig. 1: Uniformization for closed surfaces.

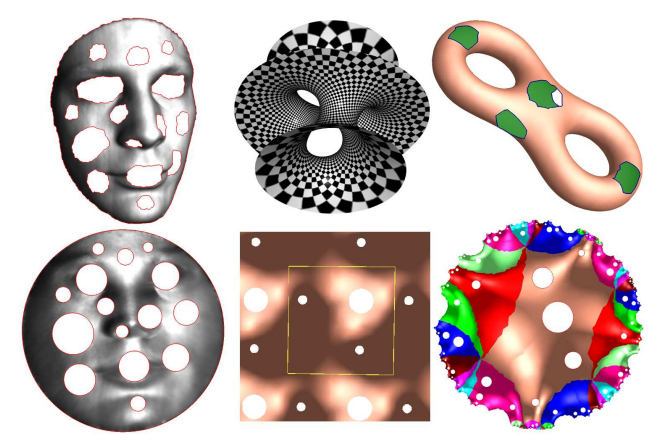

Fig. 2: Uniformization for surfaces with boundaries.

Figures 1 and 2 show the uniformizations for closed surfaces and surfaces with boundaries, respectively. The left-hand columns show the genus zero surfaces, which can conformally deform to the unit sphere with +1 curvatures. The middle columns demonstrate genus one surfaces, whose universal covering space is conformally mapped to the Euclidean plane, and the boundaries become circles. The columns on the right illustrate high genus surfaces, whose universal covering space is flattened to the hyperbolic plane, and whose boundaries are mapped to circles.

Surface uniformization can be carried out using the discrete Ricci flow algorithms [3]. Then we can compute the triangulation of the surface by performing the planar Delaunay 
refinement algorithms on the canonical uniformization domain.

\subsection{Delaunay Refinement}

The Delaunay refinement algorithm for mesh generation operates by maintaining a Delaunay triangulation, which is refined by inserting circumcenters of triangles, until the mesh meets constraints on element quality and size.

\subsubsection{Geodesic Delaunay Triangulation}

By the uniformization theorem, all oriented metric surfaces can be conformally deformed to one of three canonical shapes, the unit sphere $\mathbb{S}^{2}$, the flat torus $\mathbb{E}^{2} / \Gamma$ and the hyperbolic surface $\mathbb{H}^{2} / \Gamma$, where $\mathbb{E}^{2}$ is the Euclidean plane, $\mathbb{H}^{2}$ the hyperbolic plane, and $\Gamma$ is the Deck transformation group, a subgroup of isometries of $\mathbb{E}^{2}$ or $\mathbb{H}^{2}$, respectively. The unit sphere $\mathbb{S}^{2}$ can be conformally mapped to the complex plane by stereographic projection, with the Riemannian metric

$$
\mathbb{C} \cup\{\infty\}, \mathbf{g}=\frac{4 d z d \bar{z}}{(1+z \bar{z})^{2}} .
$$

Similarly, the hyperbolic plane $\mathbb{H}^{2}$ is represented by Poincaré's disk model with a Riemannian metric

$$
\{|z|<1 \mid z \in \mathbb{C}\}, \mathbf{g}=\frac{4 d z d \bar{z}}{(1-z \bar{z})^{2}} .
$$

The concepts of Euclidean triangles and Euclidean circles can be generalized to geodesic triangles and geodesic circles on $\mathbb{S}^{2}$ and $\mathbb{H}^{2}$. Therefore, Delaunay triangulation can be directly defined on these canonical constant curvature surfaces. A triangulation is Delaunay if it satisfies the empty circle property, namely the geodesic circumcircle of each geodesic triangle does not include any other point. Spherical circles on $\mathbb{S}^{2}$ are mapped to Euclidean circles or straight lines on the plane by stereographic projection. Similarly, hyperbolic circles are mapped to the Euclidean circles on the Poincare disk. Therefore, geodesic Delaunay triangulations on $\mathbb{S}^{2}$ or $\mathbb{H}^{2}$ are mapped to the Euclidean Delaunay triangulations on the plane. As a result, geodesic Delaunay triangulations can be carried out using the conventional Euclidean Delaunay triangulation.

\subsubsection{Delaunay Refinement on Constant Curvature Sur- faces}

The Delaunay refinement algorithm on constant curvature surfaces with empty boundary is introduced as follows. Take a flat torus $\mathbb{E}^{2} / \Gamma$ as an example. The user chooses a parameter $\varepsilon$, which is the upper bound of the circumradius.

1) An initial set of samples is generated on the surface, such that the shortest distance between any pair of samples is greater than $\varepsilon$. An initial Delaunay triangulation is constructed.

2) Select bad size triangles, whose circumradii are greater than $\varepsilon$, insert their circumcenters, and maintain the Delaunay triangulation.

3) Select bad shape triangles, whose ratio between circum radius and shortest edge length is greater than one, insert their circum centers, maintain the Delaunay triangulation.
4) Repeat 2 and 3, until the algorithm terminates.

The proof of theorem 3.3 is based on the conventional packing argument [24].

Proof: In the initial setting, all the edge lengths are greater than $\varepsilon$. In step 2, after inserting the circumcenter of a bad size triangle, all the newly generated edges are connected to the center, their lengths are no less than the circumradius, which is greater than $\varepsilon$. In step 3 , the circumradius of the bad shape triangle is greater than the shortest edge of the bad triangle, which is greater than $\varepsilon$. All the newly generated edges connecting to the center are longer than the radius $\varepsilon$. Therefore, during the refinement process, the shortest edge is always greater then $\varepsilon$.

Suppose $p$ and $q$ are the closest pair of vertices, then the line segment connecting them must be an edge of the final Delaunay triangulation, which is longer than $\varepsilon$. Therefore, the distance between any pair of vertices is greater than $\varepsilon$. Centered at the each vertex of the triangulation, a disk with radius $\varepsilon / 2$ can be drawn. All these disks are disjoint. Because the total surface area is finite, the number of vertices is finite. Therefore, the whole algorithm will terminate.

When the algorithm terminates, all triangles are well-sized and well-shaped. Namely, the circumradius of each triangle is smaller than $\varepsilon$, and the shortest edge length is greater than $\varepsilon$. For the flat torus case, the minimal angle is greater than $30^{\circ}$.

By the uniformization theorem, if a surface has a boundary, it can be conformally mapped to the constant curvature surfaces with circular holes. Then the boundaries can be approximated by the planar straight line graphs (PSLG), such that the angles between two adjacent segments are greater than $60^{\circ}$. Using a proof similar to the one given by Chew in [24] and [11], we can show the theorem still holds.

\subsubsection{Delaunay Refinement on General Surfaces}

For general surfaces, we need to add grading to the Delaunay triangulation. The grading function is the conformal factor $e^{2 \lambda}$, which controls the size of the triangles. Step 2 in the above algorithm needs to be modified as follows: select a bad size triangle with the circumcenter $p$ and circumradius greater than $\varepsilon e^{-\lambda(p)}$. The same proof can be applied to show the termination of the algorithm. In the resultant triangulation, the grading is controlled by the conformal factor, the circumradius is less than $\varepsilon e^{-\lambda}$, the shortest edge is greater than $\varepsilon e^{-\lambda}$, so the triangles are still well-shaped. On the original surface, the edge length is greater than $\varepsilon$ and the circumradius is less than $\varepsilon$. The minimal angle is bounded.

According to [47], such a kind of sampling is locally unifor$\mathrm{m}$, thus is also a $\kappa$-light $\varepsilon$-sample. Suppose the triangulation is $T, t \in T$ is a triangle, with circumradius $r(t), B \subset T$ is a union of triangles of $T$, then

Lemma 4.1: The following estimation holds

$$
\sum_{t \subset \bar{B}} r(t)^{2}+\sum_{t \subset \bar{B}, t \cap \partial B \neq \emptyset} r(t)=O(\operatorname{area}(B))+O(\text { length }(\partial B)) .
$$




\subsection{Normal Cycle Theory}

In order to be complete, we briefly introduce the normal cycle theory, which closely follows the work in [17]. For a more in-depth treatment, we refer readers to [17].

Intuitively, the normal cycle of a surface is its offset surface embedded in a higher dimensional Euclidean space. If the surface is not convex or smooth, its offset surface in $\mathbb{R}^{3}$ may have self-intersections. By embedding it in a higher dimensional space, it can be fully unwrapped.

\subsubsection{Offset Surface}

Suppose $V$ is a volumetric domain in $\mathbb{R}^{3}$, whose boundary $M=\partial V$ is a compact $C^{2}$-smooth surface. Let $\rho$ be the distance between $M$ and the medial axis of the complement of $V$. The

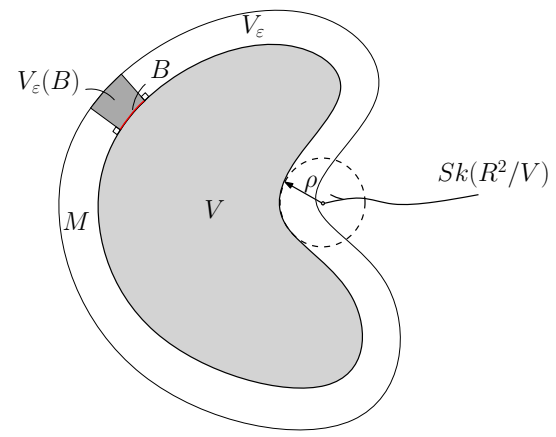

Fig. 3: Offset surface and tube formula. $\varepsilon$-offset of $V$ minus $V$ is

$$
V_{\varepsilon}=\{p \mid p \notin V d(p, V)<\varepsilon\} \subset \mathbb{R}^{3} .
$$

The tube formula can be written as

$$
\operatorname{Vol}\left(V_{\varepsilon}\right)=\operatorname{area}(M) \varepsilon+\phi_{V}^{H}(M) \frac{\varepsilon^{2}}{2}+\phi_{V}^{G}(M) \frac{\varepsilon^{3}}{3}
$$

for $\varepsilon<\rho$. The localized version of the tube formula is as follows. Let $B \subset M$ be a Borel set, the $\varepsilon$-offset of $B$ is $V_{\varepsilon}(B)$, then we have

$$
\operatorname{Vol}\left(V_{\varepsilon}(B)\right)=\operatorname{area}(B) \varepsilon+\phi_{V}^{H}(B) \frac{\varepsilon^{2}}{2}+\phi_{V}^{G}(B) \frac{\varepsilon^{3}}{3} .
$$

The volume of the $\varepsilon$-offset $V_{\varepsilon}(B)$ is always a polynomial in $\varepsilon$, and its coefficients are multiples of the curvature measures of $B$. Even if the boundary of $V$ is not smooth but if $\rho>0$, the volume of $V_{\varepsilon}(B)$ is always a polynomial in $\varepsilon$ for $\varepsilon<\rho$. Therefore the coefficients of this polynomial generalize the curvature measures from smooth surfaces to polyhedral surfaces.

This approach does not generalize to non-convex polyhedral surfaces, where $\rho$ may be equal to 0 . So the normal cycle theory has been developed. Intuitively, normal cycles provide a way of unfolding offsets in a higher dimensional space.

\subsubsection{Normal Cycles}

Definition 4.2: The normal cycle $N(M)$ of a $C^{2}$-smooth surface $M$ is the current associated with the set

$$
N(M):=\{(p, \mathbf{n}(p)) \mid p \in M\}
$$

endowed with the orientation induced by that of $M$, where a current is the generalization of an oriented surface patch, with integral coefficients. When no confusion is possible, we use the same notation $N(M)$ to denote both the current and its associated set.

The normal cycle of $V$ is the same as that of $M$, namely, $N(V)=N(M)$. The diffeomorphic mapping from $M$ to its normal cycle $N(M)$ is denoted as

$$
\begin{aligned}
i: & M \rightarrow N(M) \\
& p \rightarrow(p, \mathbf{n}(p))
\end{aligned}
$$

Suppose $V$ is a convex body, whose boundary $M$ is a

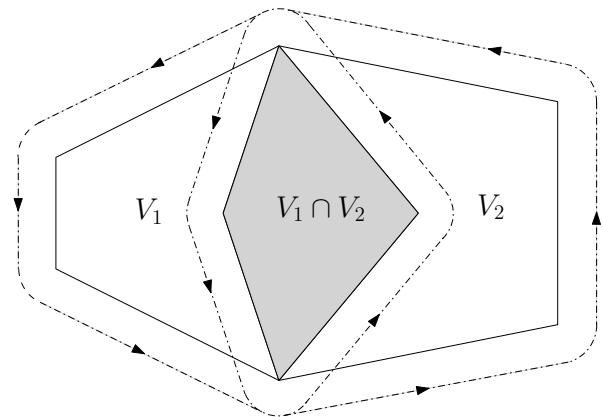

Fig. 4: Additivity of the normal cycle.

polyhedral surface. We use normal cones to replace normal vectors.

Definition 4.3: The normal cone $N C_{V}(p)$ of a point $p \in V$ is the set of unit vectors $v$ such that

$$
\forall q \in V,\langle q-p, v\rangle \leq 0 .
$$

Definition 4.4: The normal cycle of $M$ is the current associated with the set

$$
\left\{(p, \mathbf{n}(p)) \mid p \in M, \mathbf{n} \in N C_{V}(p)\right\}
$$

endowed with the orientation induced by the one of $M$. As in figure 4, normal cycles are graphically represented by their image under the map sending $(p, \mathbf{n}(p))$ to $p+\mathbf{n}(p)$.

The crucial property of the normal cycle is its additivity as shown in Fig. 4. Suppose $V_{1}$ and $V_{2}$ are two convex bodies in $\mathbb{R}^{3}$, such that $V_{1} \cup V_{2}$ is convex, then

$$
N\left(V_{1} \cap V_{2}\right)+N\left(V_{1} \cup V_{2}\right)=N\left(V_{1}\right)+N\left(V_{2}\right) .
$$

By the additivity property, we can define the normal cycle of a polyhedron. Given a triangulation of the polyhedron $V$ into tetrahedra $t_{i} . i=1,2, \cdots, n$, the normal cycle of $V$ is defined as

$$
N(V)=\sum_{k=1}^{n}(-1)^{k+1} \sum_{1 \leq i_{1}<\cdots<i_{k} \leq n} N\left(\cap_{j=1}^{k} t_{i_{j}}\right)
$$

by inclusion-exclusion. It is proved that the normal cycle $N(V)$ is independent of triangulations.

Similar to the smooth surface case, one can define a setvalued mapping from $M$ and its normal cycle $N(M)$

$$
\begin{aligned}
i: & M \rightarrow N(M) \\
& p \rightarrow(p, \mathbf{n}(p)) \quad \mathbf{n} \in N C_{V}(p) .
\end{aligned}
$$

\subsubsection{Invariant Differential 2-Forms}

Normal cycles are embedded in the space $\mathbb{R}^{3} \times \mathbb{R}^{3}$, denoted as $E_{p} \times E_{n}$, where $E_{p}$ is called point space, and $E_{n}$ is called 
normal space. Let $g$ be a rigid motion of $\mathbb{R}^{3}, g(p)=R p+d$, where $R$ is a rotation matrix, $d$ is a translation vector. $g$ can be extended to $E_{p} \times E_{n}$ as $\hat{g}(p, n)=(R(p)+d, R(n))$. We say that a differential 2-form $\omega$ is invariant under rigid motions, if

$$
\hat{g}^{*} \omega=\omega
$$

The following invariant 2-forms play fundamental roles in the normal cycle theory,

Definition 4.5: Let the coordinates of $E_{p} \times E_{n}$ be $\left(x^{1}, x^{2}, x^{3}, y^{1}, y^{2}, y^{3}\right)$, then

$$
\begin{aligned}
\omega^{A}= & y^{1} d x^{2} \wedge d x^{3}+y^{2} d x^{3} \wedge d x^{1}+y^{3} d x^{1} \wedge d x^{2} \\
\omega^{G}= & y^{1} d y^{2} \wedge d y^{3}+y^{2} d y^{3} \wedge d y^{1}+y^{3} d y^{1} \wedge d y^{2} \\
\omega^{H}= & y^{1}\left(d x^{2} \wedge d y^{3}+d y^{2} \wedge d x^{3}\right)+ \\
& y^{2}\left(d x^{3} \wedge d y^{1}+d y^{3} \wedge d x^{1}\right)+ \\
& y^{3}\left(d x^{1} \wedge d y^{2}+d y^{1} \wedge d x^{2}\right) .
\end{aligned}
$$

Curvature measures of a surface can be recovered by integrating specific differential forms on its normal cycle. The following formula unifies the curvature measures on both smooth surfaces and polyhedral surfaces. For a Borel set $B \subset \mathbb{R}^{3}$, the curvature measures are given by

$$
\begin{aligned}
\int_{N(M)} \omega_{\mid i(B \cap M)}^{G} & =\phi_{M}^{G}(B) \\
\int_{N(M)} \omega_{\mid i(B \cap M)}^{H} & =\phi_{M}^{H}(B) \\
\int_{N(M)} \omega_{\mid i(B \cap M)}^{A} & =\operatorname{area}(B)
\end{aligned}
$$

where $\omega_{\mid i(B \cap M)}^{G}$ denotes the restriction of $\omega$ to $i(B \cap M)$.

\subsection{Estimation}

In this section, we explicitly estimate the Hausdorff distance, the normal deviation, and the differences in curvature measures from the discrete triangular mesh to the smooth surface.

\subsubsection{Configuration}

Let $(M, \mathbf{g})$ be a $C^{2}$ metric surface. $\mathscr{D}$ is the unit disk on the $u v$ plane. A conformal parameterization is given by $\varphi: \mathscr{D} \rightarrow M$, such that $\mathbf{g}(u, v)=e^{2 \lambda(u, v)}\left(d u^{2}+d v^{2}\right)$. Suppose $p \in \mathscr{D}$ is a point on the parameter domain, then $\varphi(p)$ is a point on the surface. The derivative map $\left.d \varphi\right|_{p}: T_{p} \mathscr{D} \rightarrow T_{\varphi(p)} M$ is a linear map

$$
\left.d \varphi\right|_{p}=e^{\lambda(p)}\left(\begin{array}{rr}
\cos \theta & -\sin \theta \\
\sin \theta & \cos \theta
\end{array}\right) .
$$

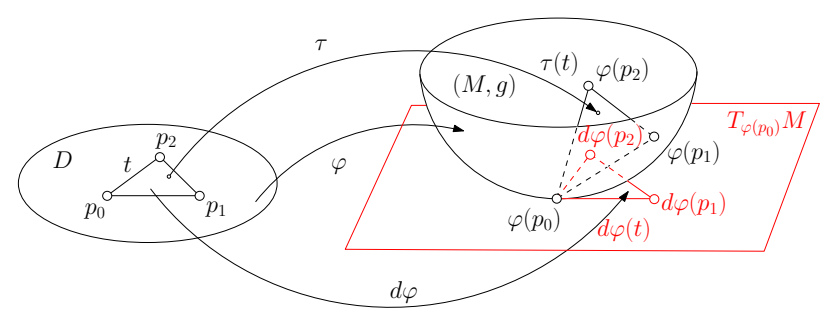

Fig. 5: Configuration.

Let $\mathscr{T}$ be a triangulation of the parameter domain $\mathscr{D}$, the conformal mapping $\varphi$ induces a triangulation $\varphi(\mathscr{T})$ of the smooth surface $M, T$ is the corresponding polyhedral surface.

\begin{tabular}{|l|l|}
\hline Symbols & Meaning \\
\hline \hline Surfaces & \\
\hline$M$ & smooth metric surface \\
$\mathscr{D}$ & parameter domain \\
\hline Mappings & \\
\hline$\varphi: \mathscr{D} \rightarrow M$ & conformal parameterization \\
$\left.d \varphi\right|_{p}: T_{p} \mathscr{D} \rightarrow T_{\varphi(p)} M$ & derivative map of $\varphi$ \\
$\tau: \mathscr{T} \rightarrow T$ & piecewise linear map between meshes \\
$\eta=\varphi \circ \tau^{-1}: T \rightarrow M$ & natural projection from $T$ to $M$ \\
$\pi: T \rightarrow M$ & closest point projection from $T$ to $M$ \\
\hline Triangulations & \\
\hline $\mathscr{T}$ & triangulation on $\mathscr{D}$ \\
$\varphi(\mathscr{T})$ & surface triangulation induced by $\varphi$ \\
$T$ & polyhedral surface induced by $\varphi(\mathscr{T})$ \\
\hline Faces & \\
\hline$t \subset \mathscr{D}$ & a triangle on parameter domain \\
$d \varphi(t) \subset T_{\varphi(p)} M$ & corresponding face on tangent plane \\
$\tau(t) \subset T$ & corresponding face on $T$ \\
\hline
\end{tabular}

TABLE 1: Symbol list

Each vertex $p_{i} \in \mathscr{T}$ corresponds to a vertex $\varphi\left(p_{i}\right) \in T$, each triangle on $\mathscr{T}$ corresponds to a face in $T$. This induces a piecewise linear map $\tau: \mathscr{T} \rightarrow T$ (see Fig. 5 and Tab. 1).

Definition 4.6 (Natural projection): The homeomorphism from the triangle mesh $T$ to the smooth surface $M$

$$
\eta=\varphi \circ \tau^{-1}: T \rightarrow M
$$

is called the natural projection.

Another map from the mesh to the surface is the closest point projection.

Definition 4.7 (Closest point projection): Suppose $T$ has no intersection with the medical axis of $M$. Let $q \in T$, and $\pi(q)$ be its closest point on the surface $M$,

$$
\pi(q)=\operatorname{argmin}_{r \in M}|r-q|,
$$

we call the mapping from $q$ to its closest point $\pi(q)$ as the closest point projection.

We will show that the closest point projection is also a homeomorphism.

\subsubsection{Hausdorff Distance and Normal Deviation}

In the following discussion, we assume the triangulation is generated by the Delaunay Refinement in Theorem 3.3. Our goal is to estimate the Hausdorff distance and the normal deviation, in terms of both the natural projection and the closest point projection.

Lemma 4.8 (Natural projection): Suppose $q \in T$, then

$$
\begin{aligned}
|q-\eta(q)| & =O\left(\varepsilon^{2}\right), \\
|\mathbf{n}(q)-\mathbf{n}(\eta(q))| & =O(\varepsilon) .
\end{aligned}
$$

Proof: As shown in Fig. 5, suppose $p \in \mathscr{D}, \tau(p)=q \cdot p$ is inside a triangle $t=\left[p_{0}, p_{1}, p_{2}\right]$,

$$
p=\sum_{k=0}^{2} \alpha_{k} p_{k}, 0 \leq \alpha_{k} \leq 1,
$$


where $\alpha_{k}$ 's are barycentric coordinates. All the edge lengths are $\Theta(\varepsilon)$, and angles are bounded. The area is $\Theta\left(\varepsilon^{2}\right)$.

Equation 8: By the linearity of $\tau$ and $d \varphi, \tau\left(p_{k}\right)=\varphi\left(p_{k}\right)$ and $\left|\varphi\left(p_{k}\right)-d \varphi\left(p_{k}\right)\right|=O\left(\varepsilon^{2}\right)$, we obtain

$$
\begin{aligned}
|\tau(p)-d \varphi(p)| & =\left|\sum_{k} \alpha_{k}\left(\tau\left(p_{k}\right)-d \varphi\left(p_{k}\right)\right)\right| \\
& \leq \sum_{k} \alpha_{k}\left|\varphi\left(p_{k}\right)-d \varphi\left(p_{k}\right)\right| \\
& =O\left(\varepsilon^{2}\right) .
\end{aligned}
$$

Therefore

$$
|\tau(p)-\varphi(p)| \leq|\tau(p)-d \varphi(p)|+|d \varphi(p)-\varphi(p)|=O\left(\varepsilon^{2}\right),
$$

where $q=\tau(p)$ and $\eta(q)=\varphi \circ \tau^{-1}(q)=\varphi(p)$, this gives Eqn. 8.

Equation 9: Construct local coordinates on the tangent plane $T_{\varphi\left(p_{0}\right)} M$, such that $\varphi\left(p_{0}\right)$ is at the origin, $d \varphi\left(p_{1}\right)$ is along the $\mathrm{x}$-axis. Then $\tau\left(p_{1}\right)$ is $\left(\Theta(\varepsilon), 0, O\left(\varepsilon^{2}\right)\right), \tau\left(p_{2}\right)$ is $\left(\Theta(\varepsilon) \cos \beta, \Theta(\varepsilon) \sin \beta, O\left(\varepsilon^{2}\right)\right)$, where $\beta$ is the angle at $p_{0}$. By direct computation, the normal to the face $\tau(t)$ is $(O(\varepsilon), O(\varepsilon), \Theta(1))$. Therefore

$$
\left|\mathbf{n} \circ \tau(p)-\mathbf{n} \circ \varphi\left(p_{0}\right)\right|=O(\varepsilon) .
$$

Furthermore,

$$
\begin{aligned}
\left|\mathbf{n} \circ \varphi(p)-\mathbf{n} \circ \varphi\left(p_{0}\right)\right| & =\left|W\left(\varphi(p)-\varphi\left(p_{0}\right)\right)\right| \\
& \leq\|W\|\left|\varphi(p)-\varphi\left(p_{0}\right)\right| \\
& =O(\varepsilon),
\end{aligned}
$$

where $W$ is the Weigarten map. $M$ is compact, therefore $\|W\|$ is bounded, $\left|\varphi(p)-\varphi\left(p_{0}\right)\right|$ is $O(\varepsilon)$.

$$
\begin{aligned}
|\mathbf{n} \circ \tau(p)-\mathbf{n} \circ \varphi(p)| & \leq\left|\mathbf{n} \circ \varphi(p)-\mathbf{n} \circ \varphi\left(p_{0}\right)\right| \\
& +\left|\mathbf{n} \circ \tau(p)-\mathbf{n} \circ \varphi\left(p_{0}\right)\right| \\
& =O(\varepsilon) .
\end{aligned}
$$

This gives Eqn. 9 .

Lemma 4.9 (Closest point projection): Suppose $q \in T$, then

$$
\begin{aligned}
|q-\pi(q)| & =O\left(\varepsilon^{2}\right), \\
|\mathbf{n}(q)-\mathbf{n}(\pi(q))| & =O(\varepsilon) .
\end{aligned}
$$

Proof: Equation 10: From Eqn. 8 and the definition of closest point, we obtain

$$
|q-\pi(q)| \leq|q-\eta(q)|=O\left(\varepsilon^{2}\right)
$$

Equation 11: From Eqn. 8 and Eqn. 10, we get

$$
|\eta(q)-\pi(q)| \leq|\eta(q)-q|+|q-\pi(q)|=O\left(\varepsilon^{2}\right),
$$

therefore

$$
|\mathbf{n} \circ \eta(q)-\mathbf{n} \circ \pi(q)| \leq\|W\||\eta(q)-\pi(q)|=O\left(\varepsilon^{2}\right) .
$$

Then from Eqn. 9 and the above equation,

$$
\begin{aligned}
|\mathbf{n}(q)-\mathbf{n}(\pi(q))| & \leq|\mathbf{n}(q)-\mathbf{n} \circ \eta(q)| \\
& +|\mathbf{n} \circ \eta(q)-\mathbf{n} \circ \pi(q)| \\
& =O(\varepsilon)+O\left(\varepsilon^{2}\right) .
\end{aligned}
$$

Remark The proofs for the Hausdorff distances in Eqn. 8 and Eqn. 10 do not require the triangulation to be well-shaped, but only well-sized. The proofs for the normal deviation

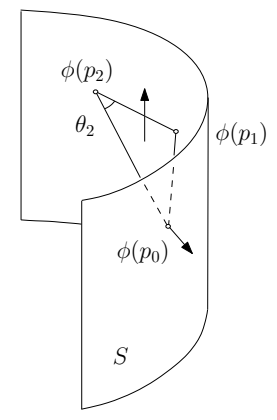

Fig. 6: Small triangles inscribed to attitudinal circles of a cylinder do not guarantee the normal convergence.

estimation in Eqn. 9 and Eqn. 11 require the triangulation to be both well-sized and well-shaped. In the proofs we use the facts that the triangulation on parameter domain has bounded angles, and the mapping $\varphi$ is conformal. Figure 6 shows a counterexample: a triangle is inscribed in a latitudinal circle of a cylinder, no matter how small it is, its normal is always orthogonal to the surface normals.

\subsubsection{Global Homeomorphism}

Both the natural projection and the closest point projection are homeomorphisms. While it is trivial for natural projection, in the following we give detailed proof to show that the closest point projection is a piecewise diffeomorphism, and we estimate its Jacobian.

Lemma 4.10: The closest point projection $\pi: T \rightarrow M$ is a homeomorphism.

Proof: First we show that $\pi$ restricted to the one-ring neighborhood of each vertex of $T$ is a local homeomorphism. Suppose $p \in T$ is a vertex, therefore $p \in M$ as well. $U(p)$ is the union of all faces adjacent to $p$. We demonstrate that $\pi$ : $U(p) \rightarrow M$ is bijective. Assume $q \in U(p)$, then $|p-q|=O(\varepsilon)$,

$$
|\pi(q)-p| \leq|\pi(q)-q|+|q-p|=O\left(\varepsilon^{2}\right)+O(\varepsilon) .
$$

Therefore

$$
|\mathbf{n}(\pi(q))-\mathbf{n}(p)|=O(\varepsilon) .
$$

Assume there is another point $r \in U(p)$, such that $\pi(q)=\pi(r)$. Let the unit vector of the line segment connecting them be

$$
\mathbf{d}=\frac{r-q}{|r-q|}
$$

then because $r, q \in U(p), \mathbf{d}$ is almost orthogonal to $\mathbf{n}(p)$,

$$
\langle\mathbf{d}, \mathbf{n}(p)\rangle=O(\varepsilon) .
$$

On the other hand, $\mathbf{d}$ is along the normal direction at $\pi(q)$, $\mathbf{n}(\pi(q))= \pm \mathbf{d}$, assume $\mathbf{d}$ is along $\mathbf{n}(\pi(q))$, from Eqn. 12, we obtain

$$
|\mathbf{d}-\mathbf{n}(p)|=O(\varepsilon) .
$$

Eqn. 13 and Eqn. 14 contradict each other. Therefore $\pi_{\mid U(p)}$ is bijective.

Then we show that $\pi$ restricted on each face is a diffeomorphism. Let $\mathbf{r}(u, v), \mathbf{n}(u, v)$ be position and normals of $M$ respectively, where $(u, v)$ are local parameters along the principal directions. $t \in T$ is a planar face. The inverse closest 
point projection map is $\pi^{-1}: \mathbf{r}(u, v) \rightarrow \mathbf{q}(u, v)$, where $\mathbf{q}(u, v)$ is the intersection between the ray through $\mathbf{r}(u, v)$ along $\mathbf{n}(u, v)$ and the face $t$,

$$
\mathbf{q}(u, v)=\mathbf{r}(u, v)+s(u, v) \mathbf{n}(u, v),
$$

direct computation shows

$$
\left\langle\mathbf{q}_{u} \times \mathbf{q}_{v}, \mathbf{n}\right\rangle=\left(1+2 H s+K s^{2}\right)\left\langle\mathbf{r}_{u} \times \mathbf{r}_{v}, \mathbf{n}\right\rangle,
$$

where $s=O\left(\varepsilon^{2}\right)$. When $\varepsilon$ is small enough, the above equation is close to 1 , which means $\pi_{|U(P)|}$ is a piecewise diffeomorphism.

Secondly, we show that $\pi$ is a global homeomorphism. We have shown that $\pi$ is a covering map. At each vertex of $T$, the closest point equals itself, therefore the degree of $\pi$ is 1 . So $\pi$ is a global homeomorphism.

Note that, the estimation of the Jacobian of the closest point projection in Eqn. 15 can be applied to show the following. Suppose $B \subset \mathbb{R}^{3}$ is a Borel set, then

$$
|\operatorname{area}(B \cap T)-\operatorname{area}(\pi(B) \cap M)|=K \varepsilon^{2} .
$$

\subsubsection{Proof of the Main Theorem}

The proof of the main Theorem 3.4. associated with the closest point projection $\pi$ is a simple corollary of the following main theorem in [17].

Theorem 4.11: Suppose $T$ is a bounded aspect ratio triangulation projecting homeomorphically on $M$, if $B$ is a relative interior of a union of triangles of $T$, then

$$
\begin{aligned}
\left|\phi_{T}^{G}(B)-\phi_{M}^{G}(\pi(B))\right| & \leq K \varepsilon \\
\left|\phi_{T}^{H}(B)-\phi_{M}^{H}(\pi(B))\right| & \leq K \varepsilon
\end{aligned}
$$

where for fixed $M$

$$
K=O\left(\sum_{\{t \in T, t \subset \bar{B}\}} r(t)^{2}\right)+O\left(\sum_{\{t \in T, t \subset \bar{B}, t \cap \partial B \neq \emptyset\}} r(t)\right),
$$

$r(t)$ is the circumradius of triangle $t$.

Proof (Closest point projection): By Lemma 4.10, the closest point projection is a homeomorphism. By Theorem 3.3, the triangulation $T$ has a bounded aspect ratio, therefore the conditions of Theorem 4.11 are satisfied, and consequently, Eqns. 16 and 17 hold. According to Eqn. 7 in Lemma 4.1, therefore the main theorem holds.

The proof of the main Theorem 3.4. associated with the natural projection $\eta$ is more direct and more adapted to our framework.

Proof (Natural projection): The natural projection $\eta$ : $T \rightarrow M$ can be lifted to a mapping between the two normal cycles $f: N(T) \rightarrow N(M)$, such that the following diagram commutes:

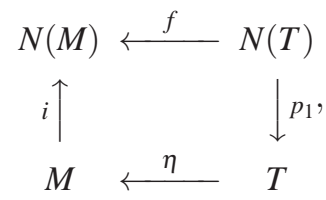

where $p_{1}$ is the projection from $E_{p} \times E_{n}$ to $E_{p}$, and $i(q)=$ $(q, \mathbf{n}(q))$ for all $q \in M$. Namely, given a point $q \in T$, and $\mathbf{n}(q)$ in its normal cone, $(q, \mathbf{n}(q)) \in N(T)$,

$$
f:(q, \mathbf{n}(q)) \rightarrow(\eta(q), \mathbf{n} \circ \eta(q)) \in N(M) .
$$

By Lemma 4.8,

$$
|(q, \mathbf{n}(q))-f(q, \mathbf{n}(q))|=O(\varepsilon) .
$$

It is obvious that $f$ is continuous.

Let $B \subset E_{p}$, we denote the current $N(T) \cap\left(B \times E_{n}\right)$ by $D$, and the current $N(M) \cap\left(\eta(B) \times E_{n}\right)$ by $E$, as shown in Fig. 7 . Consider the affine homotopy $h$ between $f$ and the identity,

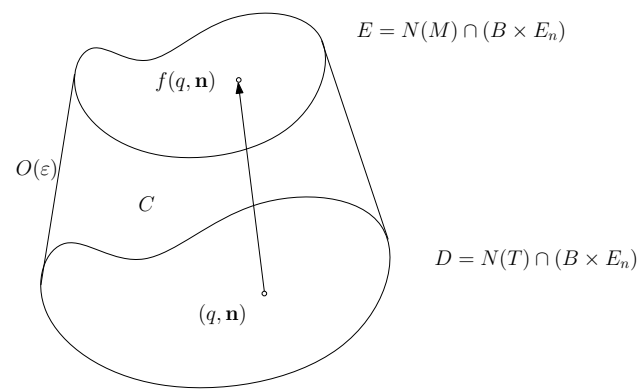

Fig. 7: Homotopy between the normal cycles $N(T)$ and $N(M)$.

$$
h(x, \cdot)=(1-x) \operatorname{id}(\cdot)+x f(\cdot), x \in[0,1] .
$$

We define the volume swept by the homotopy as

$$
C=h_{\#}([0,1] \times D),
$$

whose boundary is

$$
\partial C=E-D-h_{\#}([0,1] \times \partial D) .
$$

Intuitively, $C$ is a prism, the ceiling is $E$, the floor is $D$, and the walls are $h_{\#}([0,1] \times \partial D)$.

$$
\phi_{M}^{G}(\eta(B))-\phi_{T}^{G}(B)=\int_{E-D} \omega^{G}=\int_{\partial C} \omega^{G}+\int_{h_{\#}([0,1] \times \partial D)} \omega^{G} .
$$

By Stokes' Theorem,

$$
\int_{\partial C} \omega^{G}=\int_{C} d \omega^{G}
$$

Both $\omega^{G}$ and its exterior derivative $d \omega^{G}$ are bounded, therefore, we need to estimate the volume of block $C$ and the area of the wall $h_{\#}([0,1] \times \partial D)$. We use $\mathbf{M}(\cdot)$ to denote the flat norm (volume, area, length).

The volume of the prism $C$ is bounded by the height and the section area. The height is bounded by sup $|f-i d|$. The section area is bounded by the product of the bottom area $\mathbf{M}(D)$ and the square of the norm

$$
\|D h(x, \cdot)\|^{2}=\|x D f+(1-x) i d\|^{2} \leq(x \sup \|D f\|+(1-x))^{2} .
$$

In later discussion, we will see that $\sup \|D f\| \geq 1$, therefore

$$
\|D h(x, \cdot)\| \leq \sup \|D f\| \text {. }
$$

We obtain

$$
\begin{aligned}
\mathbf{M}(C) & \leq \mathbf{M}(D) \text { sup }|f-i d| \text { sup }\|D f\|^{2}, \\
\mathbf{M}\left(h_{\#}([0,1] \times \partial D)\right) & \leq \mathbf{M}(\partial D) \text { sup }|f-i d| \text { sup }\|D f\| .
\end{aligned}
$$

Now we estimate each term one by one.

1) Eqn. 18 shows

$$
\sup |f-i d|=O(\varepsilon) \text {. }
$$


2) Since the triangulation has a bounded ratio of circumradius to edge length, we obtain

$$
\begin{aligned}
\mathbf{M}(D) & =O\left(\sum_{t \in T, t \subset \bar{B}} r(t)^{2}\right) \\
\mathbf{M}(\partial D) & =O\left(\sum_{t \in T, t \subset \bar{B}, t \cap \partial B \neq \emptyset} r(t)\right) .
\end{aligned}
$$

Let $K$ be the summation of the two terms above. According to Lemma 4.1, $K$ is bounded by the area of $B$ and the length of $\partial B$.

3) For the estimation of $\|D f\|$, we observe that on each triangle $t \in \mathscr{D}$, the mapping $\tau$ converges to $d \varphi$, so $D f$ on each triangle converges to

$$
\left(\mathbf{r}_{u}, 0\right) d u+\left(\mathbf{r}_{v}, 0\right) d v \rightarrow\left(\mathbf{r}_{u}, \mathbf{n}_{u}\right) d u+\left(\mathbf{r}_{v}, \mathbf{n}_{v}\right) d v,
$$

where $\mathbf{r}(u, v)$ and $\mathbf{n}(u, v)$ are the position and normal vectors of the smooth surface $M,(u, v)$ the conformal parameters, namely, $\left|\mathbf{r}_{u}\right|=e^{\lambda},\left|\mathbf{r}_{v}\right|=e^{\lambda}$ and $\mathbf{r}_{u} \perp \mathbf{r}_{v}$. Assume $(d u, d v)=(\cos \theta, \sin \theta)$ for any angle $\theta$, we obtain that the norm of the tangent vector on the left hand side is $e^{\lambda}$. The norm of the vector on the right hand side is bounded by the eigenvalues of the following matrix

$$
\left[\begin{array}{cc}
\left\langle\left(\mathbf{r}_{u}, \mathbf{n}_{u}\right),\left(\mathbf{r}_{u}, \mathbf{n}_{u}\right)\right\rangle & \left\langle\left(\mathbf{r}_{u}, \mathbf{n}_{u}\right),\left(\mathbf{r}_{v}, \mathbf{n}_{v}\right)\right\rangle \\
\left\langle\left(\mathbf{r}_{v}, \mathbf{n}_{v}\right),\left(\mathbf{r}_{u}, \mathbf{n}_{u}\right)\right\rangle & \left\langle\left(\mathbf{r}_{v}, \mathbf{n}_{v}\right),\left(\mathbf{r}_{v}, \mathbf{n}_{v}\right)\right\rangle
\end{array}\right]=e^{2 \lambda} i d+I I I,
$$

where the third fundamental form is

$$
I I I=\left[\begin{array}{cc}
\left\langle\mathbf{n}_{u}, \mathbf{n}_{u}\right\rangle & \left\langle\mathbf{n}_{u}, \mathbf{n}_{v}\right\rangle \\
\left\langle\mathbf{n}_{v}, \mathbf{n}_{u}\right\rangle & \left\langle\mathbf{n}_{v}, \mathbf{n}_{v}\right\rangle
\end{array}\right] .
$$

From $I I I-2 H I I+G I=0$, where the first fundamental form $I=e^{2 \lambda} i d$, the second fundamental form $I I=e^{2 \lambda} W$, $W$ is the Weigarten matrix, we get

$$
I I I=2 H I I-G I=e^{2 \lambda}(2 H W-G i d) .
$$

Plugging into Eqn. 19, we get $\|D f\|^{2}$ bounded by the eigenvalues of

$$
(1-G) i d+2 H W,
$$

therefore on each face

$$
\|D f\|^{2} \leq \max \left\{1+k_{1}^{2}, 1+k_{2}^{2}\right\} .
$$

So $\|D f\|^{2}$ is globally bounded.

Putting all the estimates together, we obtain

$$
\left|\phi_{M}^{G}(\eta(B))-\phi_{T}^{G}(B)\right| \leq K \varepsilon .
$$

According to Lemma 4.1, $K$ is bounded by the area of $B$ and the length of $\partial B$. The proof for the mean curvature measure is exactly the same.

Remark 1. In our proofs, perfect conformality is unnecessary. All the proofs are based on one requirement: the max circumcircle of the triangles of the tessellations converge to zero. This only requires the parameterization to be K-quasiconformal, where $K$ is a positive constant, less than $\infty$.

2. It is well known that the Gauss curvature is defined on any (abstract) Riemannian surface. By the Nash theorem [48] [49], any (abstract) Riemannian surface can be isometrically embedded in a high-dimensional Euclidean space. Using the theory of normal cycle for large codimension submanifolds of Euclidean space, the inequalities (3) and (5) in Theorem 3.4 can be extended to any abstract Riemannian surface, the approximation depending on the chosen embedding.

\section{Computational Algorithm}

We verified our theoretical results by meshing spline surfaces and comparing the Gaussian and mean curvature measures.

Each spline patch $M$ is represented as a parametric smooth surface defined on a planar rectangle $\gamma: \mathscr{R} \rightarrow \mathbb{R}^{3}$, where $\mathscr{R}$ is the planar rectangle parameter domain, the position vector $\gamma$ is $C^{2}$ continuous, therefore the classical curvatures are well defined. Let $\varphi: \mathscr{D} \rightarrow M$ be the conformal mapping from the unit disk $\mathscr{D}$ to the spline surface $M$. As shown in the lefthand diagram in Diagram (20), the mapping $f$ is from $\mathscr{D}$ to $\mathscr{R}$, which makes the diagram commute, therefore $f=\gamma^{-1} \circ \varphi$.
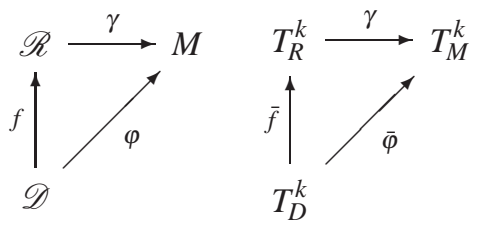

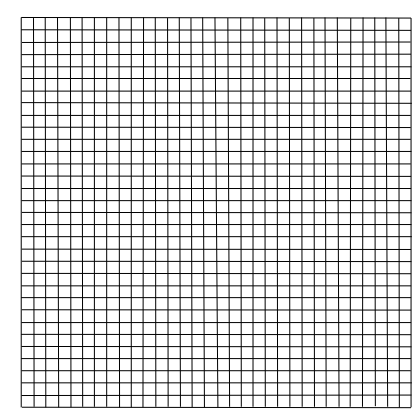

(a) regular grid $T_{R}^{0} \subset \mathscr{R}$

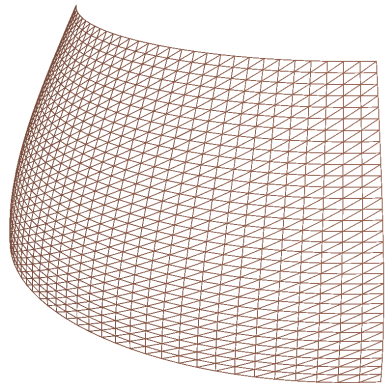

(c) $T_{M}^{0}$

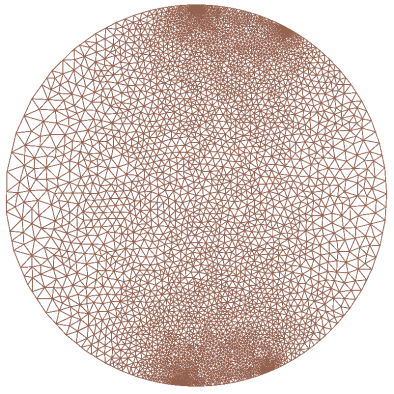

(e) Delaunay triangulation $T_{D}^{1} \subset \mathscr{D}$

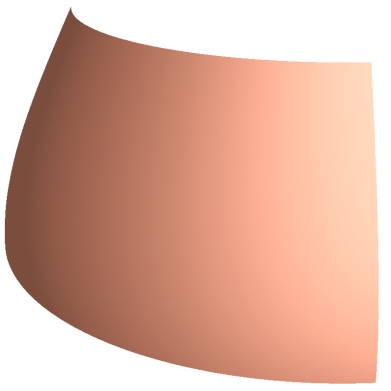

(b) $M$

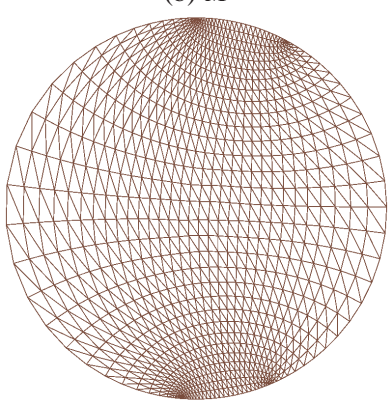

(d) $T_{D}^{0} \subset \mathscr{D}$

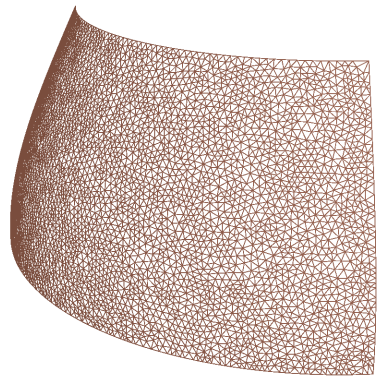

(f) $T_{M}^{1}$
Fig. 8: Pipeline for meshing a Bézier patch of Utah teapot.

As shown in Fig. 8, in our experiments, each planar domain or surface $S(S \in\{\mathscr{D}, \mathscr{R}, M\})$, is approximated by two triangle 
meshes, $T_{S}^{k}, k=0,1$, where the $T_{S}^{0}$ is induced by the regular grid on the rectangle; $T_{S}^{1}$ is induced by the Delaunay triangulation on the unit disk. Both the conformal parameterization $\varphi$ and the parameter domain mapping $f$ are approximated by piecewise linear (PL) mappings, $\bar{\varphi}$ and $\bar{f}$, respectively, which are computed on the meshes.

\subsection{Algorithm Pipeline}

\subsubsection{Conformal Parametrization}

In the first stage, the conformal parameterization is computed as follows:

$$
\bar{f}^{-1}: T_{R}^{0} \stackrel{\gamma}{\longrightarrow} T_{M}^{0} \stackrel{\bar{\varphi}^{-1}}{\longrightarrow} T_{D}^{0}
$$

$T_{R}^{0}$ is a triangulation induced by the regular grid structures on the rectangle $\mathscr{R}$. Each vertex on $T_{R}^{0}$ is mapped to the spline surface $M$ by $\gamma$, each face is mapped to a Euclidean triangle, this gives the mesh $T_{M}^{0}$. If the grid tessellation is dense, the quality of the mesh $T_{M}^{0}$ is good enough for performing the Ricci flow and we get the PL mapping $\bar{\varphi}^{-1}$, which maps $T_{M}^{0}$ to a triangulation of the disk $T_{D}^{0}$. The composition of $\bar{\varphi}$ and $\gamma^{-1}$ gives the PL mapping $\bar{f}=\gamma^{-1} \circ \bar{\varphi}: T_{D}^{0} \rightarrow T_{R}^{0}$.

\subsubsection{Resampling and Remeshing}

The process in the second stage is described in the following diagram:

$$
\bar{\varphi}: T_{D}^{1} \stackrel{\bar{f}}{\longrightarrow} T_{R}^{1} \stackrel{\gamma}{\longrightarrow} T_{M}^{1}
$$

First, we apply Ruppert's Delaunay refinement method to generate the triangulation $T_{D}^{1}$ with good quality on the unit disk. The triangulation on the disk $T_{D}^{1}$ is mapped to a triangulation $T_{R}^{1}$ on the rectangle by the PL mapping $\bar{f}: T_{D}^{0} \rightarrow T_{R}^{0}$. The connectivity of $T_{R}^{1}$ is the same as that of $T_{D}^{1}$. The vertices of $T_{R}^{1}$ are the images of the vertices of $T_{D}^{1}$ under the PL mapping $\bar{f}$, which are calculated as follows. Suppose $q$ is a Delaunay vertex of $T_{D}^{1}$ on the disk, covered by a triangle $\left[p_{0}, p_{1}, p_{2}\right] \in T_{D}^{0}$. Assume the barycentric coordinates of $q$ are $\left(\alpha_{0}, \alpha_{1}, \alpha_{2}\right), q=\sum_{k} \alpha_{k} p_{k}$, then

$$
\bar{f}(q)=\sum_{k} \alpha_{k} \bar{f}\left(p_{k}\right)
$$

The triangulation $T_{R}^{1}$ induces a triangle mesh $T_{M}^{1}$, whose connectivity is that of $T_{R}^{1}$, vertices of $T_{M}^{1}$ are the images of those of $T_{R}^{1}$ under the spline mapping $\gamma$. The discrete PL conformal mapping is given by

$$
\bar{\varphi}=\gamma \circ \bar{f}: T_{D}^{1} \rightarrow T_{M}^{1} .
$$

The triangle mesh generated by the Delaunay refinement based on conformal parameterization is $T_{M}^{1}$.

Fig. 9 shows the meshing results using the proposed method for a car model. In this experiment, the conformal parameter domain $\mathscr{D}$ is also a rectangle. Frame (a) shows a B-spline surface patch $M$; Frame (b) shows the initial triangle mesh $T_{M}^{0}$; Frame (c) shows the triangulations on the conformal parameter domain, $T_{\mathscr{D}}^{0}$ on the top and $T_{\mathscr{D}}^{1}$ at the bottom; Frames (d), (e) and (f) illustrate the triangle meshes generated by the Delaunay refinement on a conformal parameter domain with a different number of samples, $1 K, 2 K$, and $4 K$, respectively.

\section{EXPERIMENTAL Results}

The meshing algorithms are developed using generic $\mathrm{C}++$ on a Windows platform, all the experiments are conducted on a PC with Intel Core $2 \mathrm{CPU}, 2.66 \mathrm{GHz}, 3,49 \mathrm{G}$ RAM.

\subsection{Triangulation Quality}

The patch on the Utah teapot (see Fig. 8) is meshed with different sampling densities, the meshes are denoted as $\left\{T_{n}\right\}_{n=1}^{11}$ as in Tab. 2. The statistics of the meshing quality are reported in Fig. 10. Frame (a) shows the maximal circumradius of all the triangles of each mesh. Frame (b) is the average circumradius of all the triangles of each mesh. Because the sampling is uniform, we expect the circumradius $\varepsilon_{n}$ vs. the number of vertices $s_{n}$ to satisfy the relation

$$
\varepsilon_{n} \sim \frac{1}{\sqrt{s_{n}}} .
$$

The curve in Frame (b) perfectly meets our expectations. Frames (c) and (d) show the minimal angles on all meshes. According to the theory of Rupert's Delaunay refinement, the minimal angle should be no less than $20.7^{\circ}$. Frame (c) shows the minimal angles; in our experiments they are no less than $20.9^{\circ}$. Frame (d) illustrates the means of the minimal angles, which exceed $46.5^{\circ}$.

\subsection{Curvature Measure Comparisons}

For each triangle mesh $T_{k}$ produced by our method, for each vertex $\mathbf{q} \in T_{k}$, we define a small ball in $\mathbb{R}^{3}, B(\mathbf{q}, r)$ centered at $\mathbf{q}$ with radius $r$. We then calculate the curvature measures $\phi_{T_{k}}^{G}(B(\mathbf{q}, r))$ and $\phi_{T_{k}}^{H}(B(\mathbf{q}, r))$ using the formulae Eqn. 1 and Eqn. 2, respectively.

We also compute the curvature measures on the smooth surface $M, \phi_{M}^{G}(B(\mathbf{q}, r))$ and $\phi_{M}^{H}(B(\mathbf{q}, r))$ using the following method,

$$
\phi_{M}^{G}(B(\mathbf{q}, r)):=\int_{\gamma(u, v) \in B(\mathbf{q}, r)} G(u, v) g(u, v) d u d v,
$$

where $\gamma(u, v)$ is the point on the spline surface, $G(u, v)$ is the Gaussian curvature at $\gamma(u, v)$, and $g(u, v)$ is the determinant of the metric tensor. Because the spline surface is $C^{2}$ continuous, all the differential geometric quantities can be directly computed using the traditional formulas. Note that, because $M$ and $T_{k}$ are very close, we use $B(\mathbf{q}, r) \cap T_{k}$ to replace $\pi(B(\mathbf{q}, r)) \cap M$ in practice. In all our experiments, we set $r$ to be 0.05 area $(M)^{\frac{1}{2}}$ and 0.08 area $(M)^{\frac{1}{2}}$ for Gaussian and mean curvature measures, respectively.

We define the average errors between curvature measures as

$$
e_{n}^{G}=\frac{1}{\left|V_{n}\right|} \sum_{v \in V_{n}}\left|\phi_{M}^{G}(B(v, r))-\phi_{T_{n}}^{G}(B(v, r))\right|,
$$

and

$$
e_{n}^{H}=\frac{1}{\left|V_{n}\right|} \sum_{v \in V_{n}}\left|\phi_{M}^{H}(B(v, r))-\phi_{T_{n}}^{H}(B(v, r))\right|,
$$

where $V_{n}$ is the vertex set of $T_{n}$.

Figure 11 shows the errors between curvature measures with respect to sampling densities, or equivalently, the number of samples and the average circumradius. Frames (a) and (b) show that the curvature measure errors are approximately 


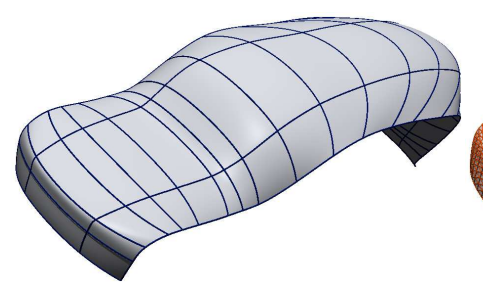

(a) spline surface $M$

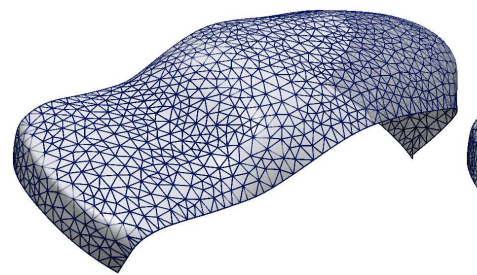

(d) Delaunay meshing result $T_{M}^{1}$ with $1 K$ samples

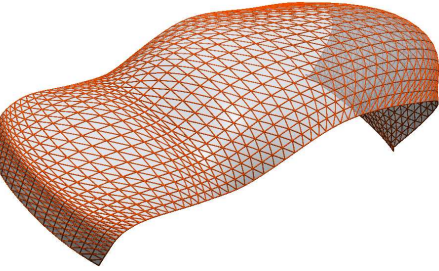

(b) initial mesh $T_{M}^{0}$

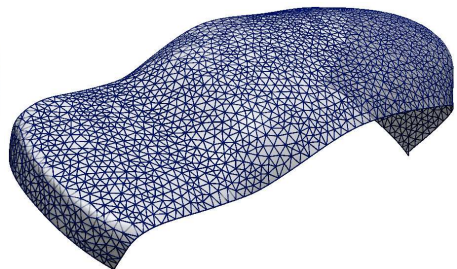

(e) Delaunay meshing result $T_{M}^{1}$ with $2 K$ samples

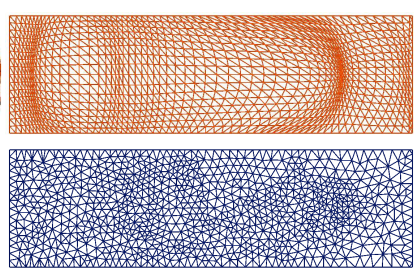

(c) triangulations on conformal parameter domain: $T_{D}^{0}$ and $T_{D}^{1}$

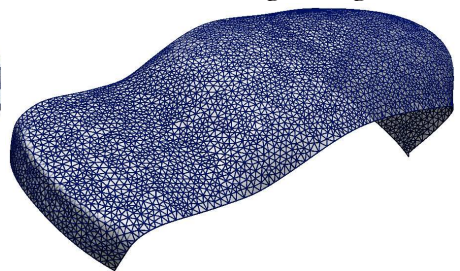

(f) Delaunay meshing result $T_{M}^{1}$ with $4 K$ samples

Fig. 9: Remeshing of the Car spline surface model.

\begin{tabular}{lrrrrrrrrrrr}
\hline mesh & $T_{1}$ & $T_{2}$ & $T_{3}$ & $T_{4}$ & $T_{5}$ & $T_{6}$ & $T_{7}$ & $T_{8}$ & $T_{9}$ & $T_{10}$ & $T_{11}$ \\
\hline \# vertex & 1021 & 2045 & 4093 & 6141 & 8189 & 10237 & 12285 & 14333 & 16381 & 18429 & 21876 \\
\# triangle & 1904 & 3910 & 7950 & 11973 & 16040 & 20118 & 24192 & 28249 & 32316 & 36372 & 43202 \\
\hline
\end{tabular}

TABLE 2: The numbers of vertices and triangles of the sequence of meshes $\left\{T_{n}\right\}$ with different resolutions.

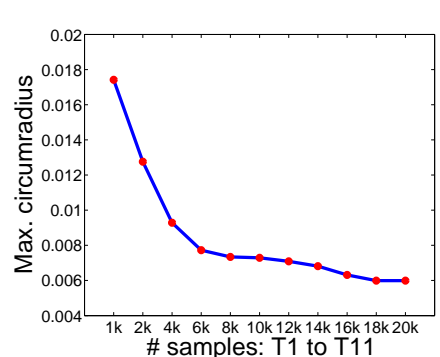

(a) $s_{n}$ vs. Max. $\varepsilon_{n}$

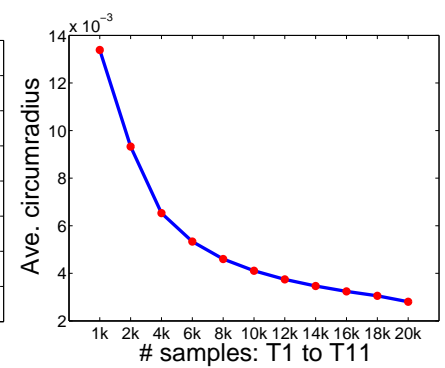

(b) $s_{n}$ vs. Ave. $\varepsilon_{n}$

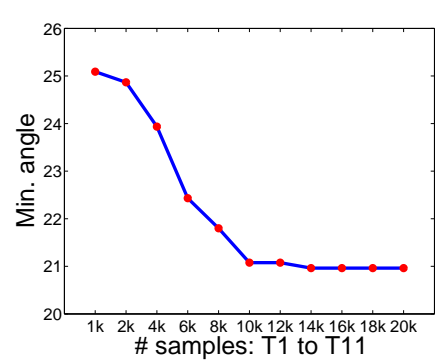

(c) $s_{n}$ vs. Min. angle

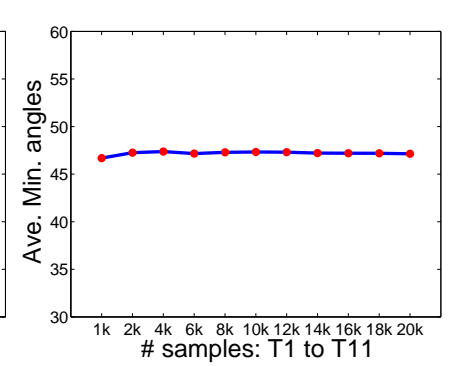

(d) $s_{n}$ vs. Ave. Min. angles

Fig. 10: The maximal and average circumradii $\left\{\varepsilon_{n}\right\}$ (a-b), and the minimal and average of minimal angles of $\left\{T_{n}\right\}$ (c-d).

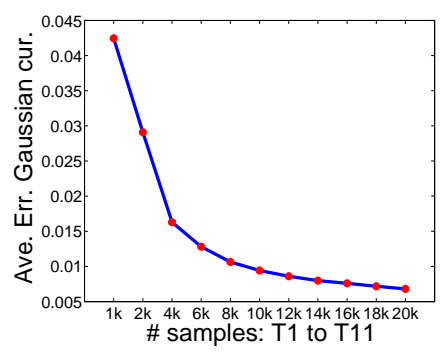

(a) $s_{n}$ vs. $e_{n}^{G}$

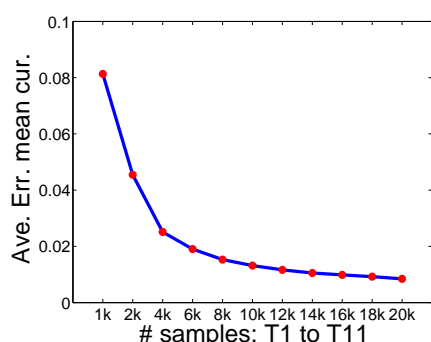

(b) $s_{n}$ vs. $e_{n}^{H}$

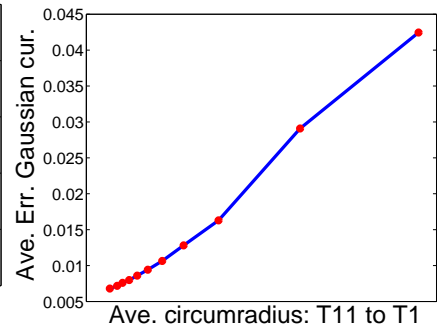

(c) Ave. $\varepsilon_{n}$ vs. $e_{n}^{G}$

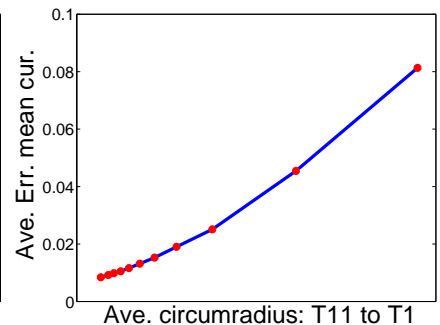

(d) Ave. $\varepsilon_{n}$ vs. $e_{n}^{H}$

Fig. 11: Curvature errors $e_{n}^{G}$ and $e_{n}^{H}$ of $\left\{T_{n}\right\}$ converge to zeros as the number of sample points goes to infinity (a-b), and as the average of the circumradii $\left\{\varepsilon_{n}\right\}$ goes to zero (c-d).

proportional to the inverse of the square root of the number of sample points; Frames (c) and (d) show the curvature measure errors are approximately linear with respect to the circumradius. This again matches our main Theorem 3.4.

Figure 12 visualizes the curvature distributions on the smooth patch $M$ (left column), and the triangle mesh $T_{11}$ (right column). The histograms show the distributions of the relative curvature errors at the vertices of the mesh. From the two left-hand columns, we can see that the curvatures of $M$ look very similar to their counterparts on $T_{11}$. Moreover, from the right-hand column, we can find that the overwhelming majority of vertices have relative curvature errors very close 


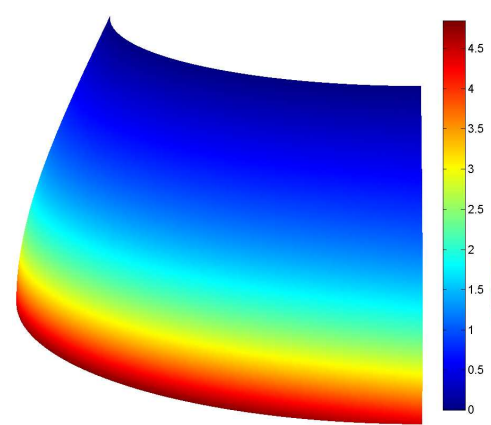

(a) Gaussian curvature of $M$

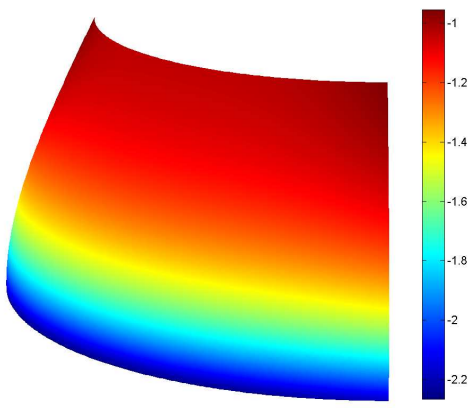

(d) mean curvature of $M$

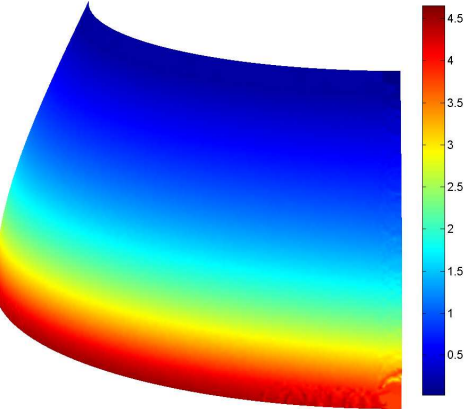

(b) Gaussian curvature of $T_{11}$

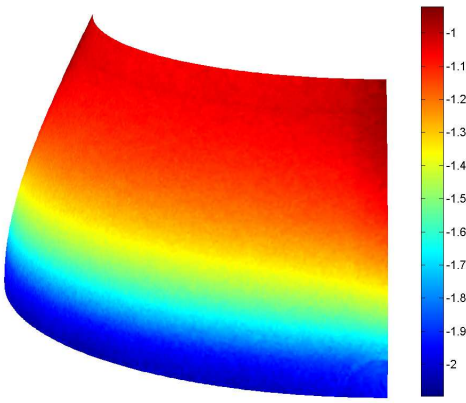

(e) mean curvature of $T_{11}$

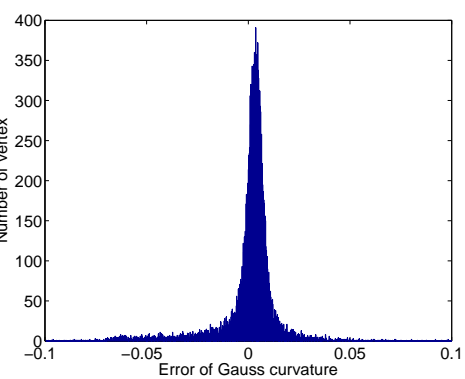

(c) histogram of Gaussian curvature error

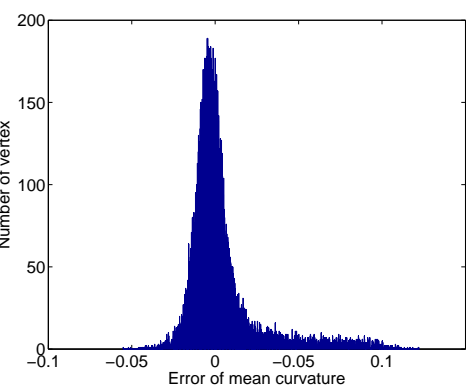

(f) histogram of mean curvature error

Fig. 12: Illustration of the curvature values on the Utah teapot spline surface patch $M,(\mathrm{a}, \mathrm{d})$, and on its approximate mesh $T_{11}$ $(b, e)$. Their relative curvature error distribution histograms are shown in (c) and (f).

to zeros. In particular, for Gaussian curvature measure, more than $97 \%$ of vertices are fall into the relative error range of $(-0.05,0.05)$. For mean curvature measure, more than $95 \%$ of vertices are included in the relative error range of $(-0.05,0.05)$. This demonstrates the accuracy of the proposed method.

\section{CONCLUSION}

This work analyzes the surface meshing algorithm based on the conformal parameterization and the Delaunay refinement method. By using the normal cycle theory and the conformal geometry theory, we rigorously prove the convergence of curvature measures, and estimate the Hausdorff distance and the normal deviation. According to [50], these theoretical results also imply the convergence of the Riemannian metric and the Laplace-Beltrami operator.

The method can be generalized to prove the curvature convergence of other meshing algorithms, such as the centroidal voronoi tessellation method, and so on. The normal cycle theory is general to arbitrary dimension. We will generalize the theoretical results of this work to include higher dimensional discretizations, such as volumetric shapes. We will explore these directions in the future.

\section{ACKNOWLEDGMENTS}

This work was supported under the grants ANR 2010 INTB 0301 01, NSF DMS-1221339, NSF Nets-1016829, NSF CCF1081424 and NSF CCF-0830550.

\section{REFERENCES}

[1] J. Sun, M. Ovsjanikov, and L. Guibas, "A Concise and Provably Informative Multi-Scale Signature Based on Heat Diffusion," Computer Graphics Forum, vol. 28, no. 5, pp. 1383-1392, 2009.
[2] Y. Wang, M. Gupta, S. Zhang, S. Wang, X. Gu, D. Samaras, and P. Huang, "High Resolution Tracking of Non-Rigid Motion of Densely Sampled 3D Data Using Harmonic Maps," Int'l Journal of Computer Vision, vol. 76, no. 3, pp. 283-300, 2008.

[3] M. Jin, J. Kim, F. Luo, and X. Gu, "Discrete Surface Ricci Flow," IEEE Transactions on Visualization and Computer Graphics, vol. 14, no. 5, pp. 1030-1043, 2008.

[4] N. Amenta and M. Bern, "Surface Reconstruction by Voronoi Filtering," in Proc. 4th Annu. Sympos. Comput. Geom., pp. 39-48, 1998.

[5] N. Amenta, S. Choi, T. K. Dey, and N. Leekha, "A Simple Algorithm for Homeomorphic Surface Reconstruction," in Proc. 6th Annu. Sympos. Comput. Geom., pp. 213-222, 2000.

[6] J.-D. Boissonnat and S. Oudot, "Provably Good Sampling and Meshing of Surfaces," Graph. Models, vol. 67, no. 5, pp. 405-451, 2005.

[7] S. W. Cheng, T. K. Dey, E. A. Ramos, and T. Ray, "Sampling and Meshing a Surface with Guaranteed Topology and Geometry," in Proc. 20th Annu. Sympos. Comput. Geom., pp. 280-289, 2004.

[8] T. K. Dey, G. Li, and T. Ray, "Polygonal Surface Remeshing with Delaunay Refinement," in Intl. Meshing Roundtable., pp. 343-361, 2005.

[9] T. K. Dey and J. A. Levine, "Delaunay Meshing of Isosurfaces.," in Proc. Shape Modeling International, pp. 241-250, 2007.

[10] J. Dai, W. Luo, M. Jin, W. Zeng, Y. He, S.-T. Yau, and X. Gu, "Geometric Accuracy Analysis for Discrete Surface Approximation," Computer Aided Geometric Design, vol. 24, no. 6, pp. 323-338, 2007.

[11] L. Paul Chew, "Guaranteed-Quality Mesh Generation for Curved Surfaces," in Proc. 9th Ann. Sympos. Computat. Geom., pp. 274-280, 1993.

[12] P. Alliez, E. C. d. Verdière, O. Devillers, and M. Isenburg, "Isotropic Surface Remeshing," in Proc. Shape Modeling International, pp. 49-59, 2003.

[13] J.-F. Remacle, C. Geuzaine, G. Compre, and E. Marchandise, "HighQuality Surface Remeshing using Harmonic Maps," Numerical Methods in Engineering, vol. 83, no. 4, pp. 403-425, 2010.

[14] E. Marchandise, C. C. de Wiart, W. G. Vos, C. Geuzaine, and J.-F. Remacle, "High-Quality Surface Remeshing using Harmonic MapsPart II: Surfaces with High Genus and of Large Aspect Ratio," Numerical Methods in Engineering, vol. 86, no. 11, pp. 1303-1321, 2011.

[15] E. Marchandise, J.-F. Remacle, and C. Geuzaine, "Quality Surface Meshing using Discrete Parametrizations," in Proc. 20th Int'l Meshing Roundtable, pp. 21-39, 2012.

[16] P. Alliez, G. Ucelli, C. Gotsman, and M. Attene, "Recent Advances in Remeshing of Surfaces," in Shape Analysis and Structuring, Mathematics and Visualization, pp. 53-82, 2008. 
[17] D. Cohen-Steiner and J. M. Morvan, "Restricted Delaunay Triangulations and Normal Cycle," in Proc. 9th Ann. Sympos. Computat. Geom., pp. 312-321, 2003.

[18] D. Cohen-Steiner and J.-M. Morvan, "Second Fundamental Measure of Geometric Sets and Local Approximation of Curvatures," J. Differential Geom., vol. 74, no. 3, pp. 363-394, 2006

[19] J. M. Morvan, Generalized Curvatures. Springer, 2008.

[20] J. M. Morvan and B. Thibert, "Approximation of the Normal Vector Field and the Area of a Smooth Surface," Discrete Computational Geometry, vol. 32, no. 3, pp. 383-400, 2004.

[21] H. Federer, Geometric Measure Theory. Springer, 1983.

[22] J. Fu, "Monge-Ampre Functions 1.," Indiana University Mathematics, vol. 38, pp. 745-771, 1989.

[23] S. Q. Xin, S. M. Chen, Y. He, G. J. Wang, X. Gu, and H. Qin, "Isotropic Mesh Simplification by Evolving the Geodesic Delaunay Triangulation," in Proc. of 8th Intl. Symposium on Voronoi Diagrams in Science and Engineering, pp. 39-47, 2011

[24] L. Paul Chew, "Guaranteed-Quality Triangular Meshes," in Department of Computer Science Tech Report 89-983, Cornell University, 1989.

[25] J. Ruppert, "A Delaunay Refinement Algorithm for Quality 2Dimensional Mesh Generation," Journal of Algorithms, vol. 18, pp. 548585, May 1995.

[26] J. R. Shewchuk, "Delaunay Refinement Algorithms for Triangular Mesh Generation," Computational Geometry: Theory and Applications, vol. 22, pp. 1-3, 2001.

[27] S.-W. Cheng, T. K. Dey, and J. R. Shewchuk, Delaunay Mesh Generation. CRC Press, 2012.

[28] Q. Du, V. Faber, and G. Max, "Centroidal Voronoi Tessellations: Applications and Algorithms," Society for Industrial and Applied Mathematics Review, vol. 41, pp. 637-676, 1999.

[29] Q. Du, G. Max, and L. Ju, "Constrained Centroidal Voronoi Tessellations for Surfaces," Society for Industrial and Applied Mathematics J. Sci. Comput., vol. 24, pp. 1488-1506, 2002.

[30] D. Yan, B. Lévy, Y. Liu, F. Sun, and W. Wang, "Isotropic Remeshing with Fast and Exact Computation of Restricted Voronoi Diagram," in Comput. Graph. Forum, pp. 1445-1454, 2009

[31] V. Surazhsky, P. Alliez, and C. Gotsman, "Isotropic Remeshing of Surfaces: A Local Parameterization Approach," in Proc. 12th Int'l Meshing Roundtable, pp. 215-224, 2003.

[32] B. Lévy and N. Bonneel, "Variational Anisotropic Surface Meshing with Voronoi Parallel Linear Enumeration," in Proc. 21st Int'l Meshing Roundtable, pp. 349-366, 2013.

[33] A. Sheffer, E. Praun, and K. Rose, "Mesh parameterization Methods and their Applications," Foundations and Trends in Computer Graphics and Vision, vol. 2, no. 2, pp. 105-171, 2006.

[34] M. S. Floater and K. Hormann, "Surface Parameterization: a Tutorial and Survey," in Advances in Multiresolution for Geometric Modelling, pp. 157-186, 2005.

[35] M. Desbrun, M. Meyer, and P. Alliez, "Intrinsic Parameterizations of Surface Meshes," Comp. Graph. Forum, vol. 21, no. 3, pp. 209-218, 2002 .

[36] X. Gu, Y. Wang, T. F. Chan, P. M. Thompson, and S.-T. Yau, "Genus Zero Surface Conformal Mapping and its Application to Brain Surface Mapping," IEEE Trans Med Imaging, vol. 23, no. 8, pp. 949-958, 2004.

[37] Y. Wang, X. Gu, K. M. Hayashi, T. F. Chan, P. M. Thompson, and S.T. Yau, "Surface Parameterization using Riemann Surface Structure," in Int'l Conf. on Computer Vision, pp. 1061-1066, 2005.

[38] B. Lévy, S. Petitjean, N. Ray, and J. Maillot, "Least Squares Conformal Maps for Automatic Texture Atlas Generation," in Proc. 29th Ann. Conf. Computer Graph. and Interactive Techniques, pp. 362-371, 2002.

[39] S. Haker, S. Angenent, A. Tannenbaum, R. Kikinis, G. Sapiro, and M. Halle, "Conformal Surface Parameterization for Texture Mapping," IEEE Trans. Visualization and Computer Graphics, vol. 6, no. 2, pp. 181-189, 2000.

[40] M. Hurdal, K. Stephenson, P. Bowers, D. Sumners, and D. Rottenberg, "Coordinate Systems for Conformal Cerebellar Flat Maps," NeuroImage, vol. 11, no. 5; PART 2, pp. 467-467, 2000.

[41] A. Sheffer and E. de Sturler, "Parameterization of Faceted Surfaces for Meshing using Angle-Based Flattening," Engineering With Computers, vol. 17 , no. 3, pp. 326-337, 2001

[42] X. Gu and S.-T. Yau, "Global Conformal Surface Parameterization," in Proc. of the Sympos. Geom. Processing, pp. 127-137, 2003.

[43] W. Zeng, D. Samaras, and X. D. Gu, "Ricci Flow for 3D Shape Analysis," IEEE Trans. Pattern Analysis and Machine Intelligence, vol. 32, no. 4, pp. 662-677, 2010.
[44] L. M. Lui, T. W. Wong, W. Zeng, X. Gu, P. M. Thompson, T. F. Chan, and S. T. Yau, "Detection of Shape Deformities Using Yamabe Flow and Beltrami Coefficients," Inverse Problems and Imaging, vol. 4, no. 2, pp. 311-333, 2010.

[45] B. Springborn, P. Schröder, and U. Pinkall, "Conformal Equivalence of Triangle Meshes," ACM Trans. Graph., vol. 27, no. 3, 2008.

[46] K. Hormann, G. Greiner, and S. Campagna, "Hierarchical Parametrization of Triangulated Surfaces," in Pro. Vision, Modeling, and Visualization, pp. 219-226, 1999.

[47] S. Funke and E. A. Ramos, "Smooth-Surface Reconstruction in Near Linear Time," in 13th ACM-SIAM Symposium on Discrete Algorithms, pp. 781-790, 2002.

[48] J. Nash, "C1 Isometric Imbeddings," Annals of Mathematics, vol. 60, no. 3, pp. 383-396, 1954

[49] J. Nash, "The Imbedding Problem for Riemannian Manifolds," Annals of Mathematics, vol. 63, no. 1, pp. 20-63, 1956.

[50] K. Hildebrandt, K. Polthier, and M. Wardetzky, "On the Convergence of Metric and Geometric Properties of Polyhedral Surfaces," Geometriae Dedicata, vol. 123, pp. 89-112, 2005

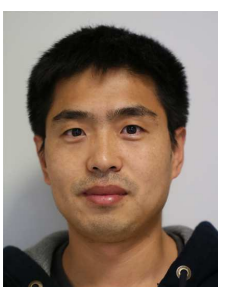

Huibin Li received a BSc degree in mathematics from Shaanxi Normal University, Xi'an, China, in 2006, and a Master's degree in applied mathematics from Xi'an Jiaotong University, Xi'an, China, in 2009. He is currently a PhD candidate in mathematics and computer science at Ecole Central de Lyon, France. His research interests include discrete curvature estimation, 3D face analysis and recognition.

Wei Zeng received her $\mathrm{PhD}$ degree from the Institute of Computing Technology, Chinese Academy of Sciences in 2008. She is an assistant professor in the School of Computing and Information Sciences, Florida International University, Miami, Florida. Her research interests include computational conformal geometry, discrete Ricci flow, and surface matching, registration, tracking, recognition and shape analysis.

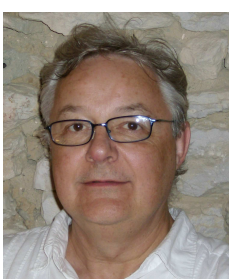

Jean Marie Morvan received his PhD from the University Paul Sabatier, Toulouse 3, France. $\mathrm{He}$ is a Professor of Mathematics, University Claude Bernard Lyon 1, France. Visiting Professor at King Abdullah University of Science and Technology, Saudi Arabia. Main interests: Differential geometry, in particular Riemannian and symplectic geometry, geometric measure theory, application of geometry to different fields such as geology, geophysics, computer graphic$\mathrm{s}$, algorithmic geometry.

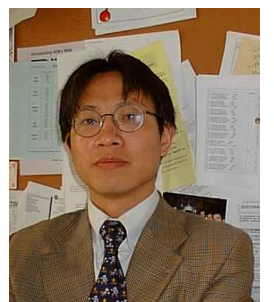

Liming Chen was awarded a joint BSc degree in Mathematics and Computer Science from the University of Nantes in 1984. He obtained a Master's degree in 1986 and a PhD in computer science from the University of Paris 6 in 1989. He first served as associate professor at the Université de Technologie de Compiègne, then joined Ecole Centrale de Lyon as Professor in 1998, where he leads an advanced research team in multimedia computing and pattern recognition. He has been Head of the department of Mathematics and Computer science from 2007. His current research interests include multimedia processing, discrete differential geometry and statistical learning, with applications in particular to 2D/3D face analysis and recognition, image and video analysis and categorization.

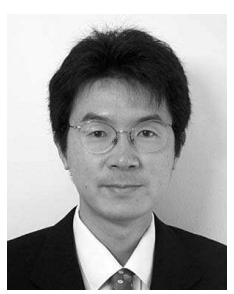

Xianfeng Gu received his $\mathrm{PhD}$ degree in Computer Science from Harvard University in 2003. $\mathrm{He}$ is an associate professor of computer science, Stony Brook University, Stony Brook, New York. His research interests include computer vision, graphics, geometric modeling and medical imaging. His major research focuses on computational conformal geometry and its applications in engineering fields. 